\title{
Muscle Metabolic Energy Costs While Modifying Propulsive Force Generation During Walking
}

Richard E. Pimentel, Noah L. Pieper, William H. Clark, and Jason R. Franz

Joint Department of Biomedical Engineering, UNC Chapel Hill and NC State University, Chapel Hill, NC, USA

\section{Corresponding Author:}

Jason Franz

jrfranz@email.unc.edu

Phone: (919) 966-6883

10206C Mary Ellen Jones Building

CB 7575

Chapel Hill, NC 27599

\section{Word Count:}

Main Text: 4934 (8151 including all captions, tables, references, and appendix)

Abstract: 100

Keywords: modeling, biofeedback, treadmill, redistribution, aging, power

\begin{abstract}
We pose that an age-related increase in the metabolic cost of walking arises in part from a redistribution of joint power where muscles spanning the hip compensate for insufficient ankle push-off and smaller peak propulsive forces $\left(\mathrm{F}_{\mathrm{P}}\right)$. Young adults elicit a similar redistribution when walking with smaller $F_{P}$ via biofeedback. We used targeted FP biofeedback and musculoskeletal models to estimate the metabolic costs of operating lower limb muscles in young adults walking across a range of Fp. Our simulations support the theory of distal-to-proximal redistribution of joint power as a determinant of increased metabolic cost in older adults during walking.
\end{abstract}

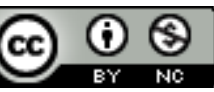

This work is licensed under a Creative Commons Attribution-NonCommercial 4.0 International License. 


\section{Introduction}

Aging and many gait pathologies are associated with reduced walking economy (i.e., higher metabolic cost when walking at the same speed). People who require greater metabolic cost to walk fatigue more quickly and are thereby likely to walk less. The functional consequences of higher metabolic cost include reduced physical activity, independence, and thereby quality of life (Morris \& Hardman 1997). Moreover, this barrier to accessible exercise disproportionally impacts populations already at increased risk for secondary health concerns such as cardiovascular disease and diabetes (World Health Organization 2019). Preserving and/or restoring walking ability can be transformative in improving physical activity and overall health (Morris \& Hardman 1997). Identifying the mechanistic determinants of higher metabolic cost of walking due to age and gait pathology is a critical first step toward translational opportunities to preserve and/or restore economical walking patterns in people with walking disability.

The higher metabolic cost of walking due to aging, in particular, is likely to affect a significant and ever-increasing proportion of our population. Older adults consume oxygen 20$30 \%$ faster than younger adults while walking at the same speed (Peterson \& Martin 2010; Hortobágyi et al. 2011). The reasons for this higher metabolic energy cost are not fully understood. Training interventions shown to improve strength and/or balance in older adults have been generally unable to reduce the metabolic cost of walking (Mian et al. 2007; Franz 2016). Several plausible explanations for this increased metabolic cost of walking have emerged and align with the neurological, muscular, and physiological changes due to aging. Some explanations, including higher metabolic costs to maintain lateral stability or to perform more total mechanical work during walking, have been refuted (Ortega \& Farley 2007; Ortega et al. 2008). Others, such as increased antagonist leg muscle coactivation in older adults, leave roughly two-thirds of the agerelated increase in metabolic cost unexplained (Peterson \& Martin 2010; Hortobágyi et al. 2011).

Biomechanically, older adults walk with a hallmark redistribution of muscular workload wherein muscles spanning the hip during early stance (i.e., hip extensors) and/or late stance and early swing (i.e., hip flexors) increase their output presumably to compensate for reduced ankle push-off - so described as a distal-to-proximal redistribution of muscular demand (Kerrigan et al. 1998; DeVita \& Hortobagyi 2000; Silder et al. 2008; Cofré et al. 2011; Boyer et al. 2017). At the limb-level, this reduced ankle push-off in older adults is accompanied by smaller peak propulsive ground reaction forces $\left(\mathrm{F}_{\mathrm{P}}\right)$ - an important determinant of walking speed (Boyer et al. 2017). This likely leads to a reduction in their preferred walking speed, which is a proxy for functional ability, mortality, and cognitive decline, with many health experts suggesting that walking speed may be a vital sign of health status of older adults (Hardy et al. 2007; Kikkert et al. 2016; Jee et al. 2019). Growing indirect evidence suggests that a distal-to-proximal redistribution of muscular workload during walking may come with a significant metabolic penalty rooted in inter-muscular differences in muscle-tendon architecture. Specifically, with their short muscle fibers and long, compliant series elastic tendons, we (Fickey et al. 2018; Browne \& Franz 2019) and others (Sawicki et al. 2009; Huang et al. 2015) have suggested that muscle-tendon units spanning the ankle are far more economical for force generation in walking than those spanning the hip, which have much longer muscle fibers and relatively shorter and less compliant tendons. However, despite its potential relevance to walking economy in aging and gait pathology, this theory has not yet been supported by direct experimental manipulation or measurement.

It is challenging to experimentally isolate hallmark features of elderly gait such as the distal-to-proximal redistribution and/or reduced Fp. Recently, our group showed that young adults walking with smaller than normal $F_{P}$ in a targeted biofeedback paradigm redistribute muscle 
workload from the ankle to the hip without changing total positive mechanical work, thereby emulating the walking biomechanics of older adults (Browne \& Franz 2017a; Fickey et al. 2018). Thus, using this biofeedback paradigm in young adults would allow for testing how differences in the relative reliance on distal versus proximal leg muscles that typically accompany reductions in $\mathrm{F}_{\mathrm{P}}$ affect muscle metabolic cost. Additionally, a young adult demographic allows for biomechanical investigation that is independent of other age-related changes that may affect metabolism (sarcopenia, tendon compliance, mitochondrial physiology, etc.).

It is not feasible to directly measure the metabolic cost of operating individual muscles during walking. Fortunately, models of the human musculoskeletal system allow for simulation of body movements and estimation of muscle dynamics underlying those movements. Thus, our purpose was to use a targeted biofeedback paradigm and musculoskeletal simulations to estimate the metabolic costs of operating lower limb muscles in young adults walking across a range of $\mathrm{F}_{\mathrm{P}}$. We first hypothesized that the simulated muscle metabolic energy cost of walking would increase in a manner consistent with whole-body measurements when targeting smaller or larger $\mathrm{F}_{\mathrm{P}}$. We also hypothesized that the metabolic costs of operating proximal versus distal leg muscles would exhibit fundamentally different responses to targeting smaller and larger FP. Ultimately, we seek to provide muscle-level insight to inform movement scientists, rehabilitation engineers, and clinicians interested in understanding and mitigating age- and disease-related increases in walking metabolic cost.

\section{Methods}

\section{Participants}

We recruited 12 young adults (7 females; age $=23.3 \pm 3.14$ years; height $=1.74 \pm 0.12 \mathrm{~m}$; mass $=74.7 \pm 14.3 \mathrm{~kg}, \mathrm{BMI}=24.6 \pm 3.0 \mathrm{~kg} / \mathrm{m}^{2}$, mean \pm standard deviation) via word of mouth to participate in this single-visit study. All participants confirmed absence of neurologic impairments and musculoskeletal injuries in the previous 6 months. Prior to data collection, each participant provided written informed consent and the study was approved by the University of North Carolina Biomedical Sciences Institutional Review Board.

\section{Experimental Protocol}

We measured each participant's preferred over-ground walking speed via the average time from three passes down a 30-meter walkway (average preferred walking speed: $1.37 \pm 0.15 \mathrm{~m} / \mathrm{s}$ ). For motion capture measurements, we placed 19 retroreflective markers on the sacrum and bilateral anterior superior iliac spines, posterior superior iliac spines, lateral/medial femoral epicondyles, lateral/medial malleoli, lateral calcanei, and lateral first and fifth metatarsal heads. An additional 14 markers tracked the thighs and shanks of each participant on rigid clusters. After a static calibration trial, we located functional hip joint centers via leg circumduction tasks to improve model scaling accuracy (Cappozzo 1984; Bell et al. 1990; Piazza et al. 2001). Prior to walking measurements, participants stood still for 5 minutes to estimate standing metabolic rate (see Metabolic Measurements). Participants then completed all walking trials at their preferred speed on a force-sensing, dual-belt treadmill (Bertec Corp., Columbus, Ohio, USA), beginning with a 5-minute warm up. The average peak anterior ground reaction force from all steps taken during the final two minutes of the warm-up determined each subjects' habitual Fp using a custom Matlab (MathWorks, Natick, MA, USA) script that extracted and analyzed ground reaction forces in real-time. 
Subjects then participated in a visual biofeedback paradigm in which they were encouraged to push off the ground with varying vigor to target their typical walking $\mathrm{F}_{\mathrm{P}}$ (Norm) as well as $\pm 20 \%$ and $\pm 40 \%$ of Norm as outlined below (Figure 1A). Using a Matlab script and visual interface described in detail previously (Browne \& Franz 2017a), a monitor positioned in front of the participant simultaneously displayed the respective target $F_{P}$ value for each trial and the average Fp from their previous 4 steps ( 2 right and 2 left), updated on a step-by-step basis (Browne \& Franz 2017a). For all trials involving biofeedback, we normalized the scaling of each subject's FP data on the monitor to evenly distribute all target values over the ordinate range. Prior to beginning the targeting biofeedback, each participant completed an exploration period to become familiarized with increasing and decreasing their $F_{P}$ across the range of target values. Then, across five separate 5-minute trials, participants walked at their preferred speed and targeted each of the $\mathrm{F}_{\mathrm{P}}$ targets in randomized order. During the final two minutes of each trial, we recorded bilateral lower body kinematics using a 12-camera motion capture system (Motion Analysis Corporation, Santa Rosa, CA, USA) operating at $100 \mathrm{~Hz}$ and measured split-belt treadmill ground reaction forces at 1000 Hz. We filtered marker trajectories and ground reaction forces using a $4^{\text {th }}$-order low-pass Butterworth filter with 6 and $20 \mathrm{~Hz}$ cutoff frequencies, respectively.

\section{Metabolic Measurements}

For this study, we repurposed metabolic measurements from a previously published study using methods therein described in detail (Pieper et al. In Revision). Briefly, expired oxygen and carbon dioxide were sampled on a breath-by-breath basis via COSMED K5 wearable metabolic system (COSMED, Rome, Italy). To estimate standing and walking metabolic rates, we averaged expired gases over the final two minutes of the 5-minute standing condition and each 5-minute walking condition, respectively. We used standardized regression equations (Brockway 1987) to estimate whole-body metabolic power from oxygen consumption and carbon dioxide production, subtracted standing from walking to calculate net metabolic power, and finally normalized outcomes by body mass $(\mathrm{W} / \mathrm{kg})$.

\section{Inclusion of Trunk Kinematics}

Our original walking data was derived from another study (Pieper et al. In Revision) and did not include trunk marker trajectories; however, the reliability of musculoskeletal simulations improves when including trunk kinematics for center of mass estimates and dynamic consistency. Thus, we opted to record and benchmark normal pelvis and trunk marker trajectories (adding sternal notch and bilateral acromion processes) in three additional participants walking at $1.2 \mathrm{~m} / \mathrm{s}$. We then used the resultant average kinematics to create a virtual trunk segment for each participant in our primary experiment. Specifically, we synchronized the measurements using timenormalized walking motions and defined virtual trunk marker locations as position vectors in the pelvis coordinate system. We scaled the position vector magnitudes to each participant's average leg length to account for inter-individual differences in subject stature. Supplementary Figure S1 shows the profiles of virtual trunk kinematics across all subjects and conditions.

\section{Musculoskeletal Modeling and Simulation}

We performed all musculoskeletal modeling and bioenergetic simulations using filtered marker trajectories and ground reaction forces in OpenSim (version 4.0) (Delp et al. 2007; Seth et al. 2018) and the gait2392 musculoskeletal model (Figure 1B). We preserved all default muscle parameters, except maximum isometric force, which was set to 1.5 times default for all models (in accordance with OpenSim documentation, and similarly performed by other groups (Arnold et al. 2013)). Subject-specific models were scaled using a combination of anatomical, functional, and 
virtual markers that aligned with the local coordinate axes of each body segment. We locked the subtalar and metatarsal-phalangeal joints at $0^{\circ}$ for all simulations because our marker set was insufficient to yield those kinematics (Rajagopal et al. 2016).

We analyzed the first left and right strides from the 10 -second window with the most accurate biofeedback performance (i.e., steps where their actual $F_{P}$ closely matched the target) for each walking condition and subject. From these two strides, inverse kinematics determined segment kinematics that minimized the sum of the square marker trajectory error. Then, we iteratively applied a residual reduction algorithm to identify model segment mass distributions and joint kinematics consistent with measured ground reaction forces. We updated model segment masses after the first iteration and used kinematics from the second iteration to ensure dynamic consistency. After residual reductions, we used computed muscle control without constraints to determine the lower limb muscle activations necessary to drive the scaled model to match the recorded kinematics.

Finally, we determined our primary outcome measure (muscle metabolic power) using two different muscle metabolic cost models (i.e., "probes") readily available within OpenSim (Umberger et al. 2003; Bhargava et al. 2004; Umberger 2010). Our purpose was not to objectively compare the models' efficacy; rather, we included two models with checks for consistency to build confidence in our model predictions. Each model estimated metabolic power for each individual muscle actuator as a function of heat rate (activation, shortening/lengthening, force maintenance, basal) and mechanical work rate (Umberger et al. 2003; Bhargava et al. 2004; Umberger 2010). It is not within the scope of this paper to discuss these models in detail. Nevertheless, each was governed by time-dependent variables that included, for each individual muscle actuator, its excitation and activation, relative $\%$ of fast versus slow twitch muscle fibers, contractile velocity, force-generating capacity, mass, and optimal fiber length.

\section{Data Reduction and Statistical Analysis}

We integrated individual muscle metabolic powers over select phases of the gait cycle (i.e., leading limb double support, single support, trailing limb double support, swing phase, and whole stride) which were calculated from instances where the vertical ground reaction force crossed a threshold of $20 \mathrm{~N}$. In addition, we normalized all metabolic outcomes by body mass and total stride time to estimate metabolic power per unit second of walking. We categorized joint-level metabolic powers as those associated with operating the hip, knee, and ankle joints during each gait cycle phase by summing individual muscle metabolic powers for actuators spanning each respective joint. We partitioned biarticular muscles into their respective joints using the ratio of their sagittal plane moment arms in a standing position (Miller 2014). Finally, we averaged individual muscle metabolic outcomes bilaterally for subsequent statistical analysis.

Pearson correlations tested for associations between measured and model-predicted metabolic powers. One-way repeated measures analyses of variance (ANOVA) tested for main effects of biofeedback condition on joint-level metabolic powers within each gait cycle phase of interest. When a significant main effect was found, Fisher's least significant difference (LSD) post-hoc tests identified pairwise differences compared to Norm. One-dimensional statistical parametric mapping (SPM, http://www.spm1d.org/, (Pataky 2012)) identified regions of the gait cycle over which individual muscle metabolic powers during targeted biofeedback conditions differed from Norm (repeated measures ANOVA with LSD post-hoc tests). We performed correlations and ANOVAs in Matlab, and calculated SPM using Python. We used a critical alpha value of 0.05 to define significance of ANOVAs. We additionally report effect sizes of partial eta 
square $\left(\eta_{\mathrm{p}}{ }^{2}\right)$ for the variance associated with main effect across biofeedback conditions as well as Cohen's d for effect size pairwise comparisons between a specific condition versus Norm.

\section{Results}

\section{Empirical Measurements versus Model Predictions}

Compared to walking normally, measured net metabolic power increased by an average of $58 \%$ and $47 \%$ when targeting $-40 \%$ and $+40 \% \mathrm{FP}$, respectively (Figure $2 \mathrm{~A}, \mathrm{p}<0.001, \eta_{\mathrm{p}}{ }^{2}=0.350$ ). Total model-predicted metabolic power followed a similar trend (Figure $2 \mathrm{~B}-\mathrm{C}$ ) and positively correlated with empirical measurements (Umberger: $\mathrm{r}^{2}=0.309, \mathrm{p}<0.001$; Bhargava: $\mathrm{r}^{2}=0.342$, $\mathrm{p}<0.001$, Figure 2E-F). These two metabolic models were strongly correlated with each other $\left(r^{2}=0.981, p<0.001\right.$, Figure 2D) and displayed similar stride-averaged profiles (Figure 2G). Given the high level of consistency between model predictions (Figure 2E, F, G), we opted to use the Umberger model for subsequent joint- and muscle-level analysis.

\section{Joint-Level Model Predictions}

Figure 3 summarizes model-predicted metabolic power integrated across periods of interest for all muscles (A) and summed for muscle actuators spanning the hip (B), knee (C), and ankle (D) joints. Due to the many statistical comparisons for the joint-level analysis, please reference Table 1 where we document the statistical outcomes of all comparisons. Here, we report only mean pairwise differences for brevity. When targeting $40 \%$ smaller $\mathrm{FP}$, metabolic power across the entire stride increased in total $(+0.79 \mathrm{~W} / \mathrm{kg})$ due to increases at the hip $(+0.29 \mathrm{~W} / \mathrm{kg})$ and $\mathrm{knee}(+0.22$ $\mathrm{W} / \mathrm{kg}$ ). These increases were mainly attributed to increased power consumed during leading leg double support at the hip $(+0.16 \mathrm{~W} / \mathrm{kg})$ and during leg swing in total $(+0.42 \mathrm{~W} / \mathrm{kg})$ and at the knee $(+0.11 \mathrm{~W} / \mathrm{kg})$. Also, when targeting $40 \%$ smaller $\mathrm{F}_{\mathrm{P}}$, ankle metabolic power increased during single support $(+0.07 \mathrm{~W} / \mathrm{kg})$ but decreased during trailing limb double support $(-0.15 \mathrm{~W} / \mathrm{kg})$, resulting in no change over the entire stride.

When targeting $40 \%$ larger $\mathrm{F}_{\mathrm{P}}$, metabolic power across the entire stride increased in total $(+0.90 \mathrm{~W} / \mathrm{kg})$ due to increases at the hip $(+0.20 \mathrm{~W} / \mathrm{kg})$, knee $(+0.11 \mathrm{~W} / \mathrm{kg})$, and ankle $(+0.10$ $\mathrm{W} / \mathrm{kg}$ ). These increases over the entire stride were primarily due to higher metabolic power during single support across all lower limb joints (hip: $+0.13 \mathrm{~W} / \mathrm{kg}$, knee: $+0.13 \mathrm{~W} / \mathrm{kg}$, ankle: +0.09 $\mathrm{W} / \mathrm{kg}$ ). We found no differences in metabolic power during swing phase when targeting $40 \%$ larger FP. However, when targeting $20 \%$ larger $\mathrm{FP}_{\mathrm{P}}$, swing phase metabolic power increased in total $(+0.11$ $\mathrm{W} / \mathrm{kg})$ and at the hip $(+0.08 \mathrm{~W} / \mathrm{kg})$. Finally, there was a reduction in metabolic cost during leading limb double support when targeting $+20 \% \mathrm{FP}$ in total $(-0.14 \mathrm{~W} / \mathrm{kg})$ and at the hip $(-0.05 \mathrm{~W} / \mathrm{kg})$ and knee $(-0.04 \mathrm{~W} / \mathrm{kg})$, which were the only significant reductions in metabolic cost across all conditions.

\section{Individual Muscle-Level Model Predictions}

Figure 4 shows the metabolic power estimated for 21 lower body muscles that contribute most to total model-predicted metabolic power during walking and the results of our statistical parametric mapping. Although there were several instances containing within-subject condition main effects, only a handful of significant pairwise differences arose and nearly all were for the $40 \%$ smaller Fp condition. Specifically, when targeting $40 \%$ smaller $\mathrm{F}_{\mathrm{P}}$, we observed the following changes in metabolic power (organized from distal to proximal): soleus consumed less during push-off; biceps femoris (long head) and semimembranosus consumed more during early single support; the vastii muscles consumed less during weight acceptance and more during mid-swing; and the gluteus medius and maximus both consumed less during push-off and more during late 
swing/early stance. When targeting $20 \%$ smaller $\mathrm{F}_{\mathrm{P}}$, we found that the vastus medialis and intermedius consumed more metabolic power during mid-swing. Finally, when targeting $40 \%$ larger $\mathrm{F}_{\mathrm{P}}$, the adductor longus consumed less metabolic power during early single support.

\section{Step Kinematics}

To provide context for our metabolic outcomes, Table 2 displays timing information for gait cycle periods of interest. Participants took longer (shorter) steps when target larger (smaller) than normal $\mathrm{F}_{\mathrm{P}}$, respectively. In relative terms (i.e., \% stride), participants spent more time in single support and leg swing and less time in double support when targeting larger $\mathrm{F}_{\mathrm{P}}$

\section{Discussion}

This study sought to provide new insight into the biomechanical basis for reduced walking economy due to age and gait pathology, and thereby inform movement scientists, rehabilitation engineers, and clinicians interested in mitigating those changes to improve independence. We accept our first hypothesis that model-predicted metabolic costs increased in a manner consistent with those measured across conditions via indirect calorimetry. We also accept our second hypothesis that proximal and distal leg muscles would exhibit different responses when targeting smaller and larger than normal $F_{P}$. Ultimately, our experimental manipulations and musculoskeletal simulations provide a muscle-level roadmap for better understanding the metabolic consequences of walking with: $(i)$ smaller than normal $\mathrm{FP}_{\mathrm{P}}$, common in aging and gait pathology, and (ii) larger than normal $\mathrm{FP}$, a therapeutic milestone for gait retraining or wearable assistive devices. More immediately, our results provide convincing evidence to suggest that a distal-to-proximal redistribution of muscle workload during walking may exact a significant metabolic penalty.

In their seminal papers, Bhargava et al. (2004) reported model predictions that tended to overestimate measured net metabolic power, while Umberger et al. (2003) and Umberger (2010) yielded moderately better agreement (Umberger et al. 2003; Bhargava et al. 2004). More recently, Koelewijn et al. (2019) found very strong positive correlations between the output from both these models and measured net metabolic power during walking $\left(\mathrm{r}^{2}=0.92\right.$ and 0.86 , respectively) (Koelewijn et al. 2019). In this study, our model predictions explained approximately one-third of the variance in empirical measurements across a relatively broad combination of walking conditions. When averaged across conditions, our estimates agreed quite well with measured values in absolute terms. However, we note two explanations for the variance unaccounted for across conditions. Specifically, our models tended to overestimate net metabolic power during normal walking while underestimating the sensitivity therein to prescribed changes in Fp. Moving forward, we anticipate refining our predictions based on higher fidelity measurements (i.e., including trunk, subtalar, and metatarsal-phalangeal joint kinematics) and improved simulation specificity (i.e., personalized "tuning" of muscle-tendon architecture (Handsfield et al. 2014) and material properties (Uchida et al. 2016; Orselli et al. 2017)). Nevertheless, because we were able to estimate changes in whole-body metabolic cost consistent with those measured, we have confidence in using musculoskeletal modeling to answer questions related to the metabolic consequences of altered muscular demand across the lower limbs during walking.

As context for our discussion of metabolic outcomes, we do note variations in step kinematics between $F_{P}$ conditions (Table 2), with shorter and faster steps when targeting smaller $F_{P}$ and vice versa. Such changes in step kinematics are known to affect metabolic cost (Donelan et al. 2002; Kuo et al. 2005; Soo \& Donelan 2010). Because the purpose of our study was to assess the metabolic costs of walking while controlling for speed, we cannot rule out the interaction between step length/duration and walking dynamics that govern our model estimates. However, 
we also know from our prior work that although both change simultaneously in response to our biofeedback paradigm, modifying $\mathrm{F}_{\mathrm{P}}$ is not biomechanically equivalent to modifying step lengths (Browne \& Franz 2017a). Hereafter, we consider metabolic costs at the joint- and muscle-level foremost as those due to changes in $\mathrm{F}_{\mathrm{P}}$, but also in the context of these changes in gait event timing.

\section{Targeting smaller than normal $F_{P}$}

Reduced ankle power generation during push-off, characteristic of aging and many gait pathologies and associated with reduced $F_{P}$, requires compensatory increases in hip mechanical output. In prior studies, this compensation has manifested as an increased demand on leading limb hip extensors to accelerate the body forward and upward (DeVita \& Hortobagyi 2000; Silder et al. 2008; Cofré et al. 2011), and on trailing limb hip flexors to initiate leg swing (Judge et al. 1996; Silder et al. 2008; Cofré et al. 2011). Our results provide evidence that such compensations have metabolic consequences. First, as expected for walking with reduced push-off intensity, we found that targeting smaller FP yielded lower metabolic costs of operating muscles spanning the ankle during trailing limb double support. More proximally, we found that increases in the metabolic cost of walking with smaller FP were well-explained by muscles spanning the hip during early stance - namely, the gluteus maximus and the biarticular hamstrings. We did not find a significant increase in metabolic cost at the hip during late stance when targeting smaller F. However, at the individual muscle level, we did find higher metabolic costs of operating the psoas and iliacus during late stance. This outcome is consistent with increased demands for swing initiation, with metabolic consequences continuing into mid-swing, where we found a large increase in metabolic cost for all four quadriceps muscles. We interpret this increase to suggest that insufficient ankle power output exacts a metabolic penalty requiring additional effort for leg swing and knee extension in preparation for each subsequent foot strike. Together, these data provide an individual muscle roadmap to explain how the distal-to-proximal redistribution increases the metabolic cost of walking.

While walking with $40 \%$ smaller $\mathrm{F}_{\mathrm{P}}$, we were surprised to find an increased metabolic cost of operating muscles spanning the ankle during single support. This increased utilization of ankle extensor muscles during single support may be due to a shift in the relative timing of the anterior GRF, which rose and peaked sooner for this biofeedback condition relative to normal walking (data not reported). At the individual muscle level, we were also surprised to find a reduced metabolic cost of operating the gluteus medius and minimus during push-off when targeting $40 \%$ smaller than normal F. The gluteus medius and minimus are generally considered important during swing for regulating foot placement to preserve lateral stability. However, some authors have recently suggested that push-off intensity and lateral balance are inextricably connected (Kim \& Collins 2015; Reimann et al. 2018). Our results are consistent with those conclusions and implicate the gluteus medius (and to a lesser extent, gluteus minimus) in providing hip stability that is proportional to push-off intensity, likely allowing for effective force transmission to the center of mass.

\section{Targeting larger than normal $F_{P}$}

The goal of many rehabilitation paradigms and wearable assistive devices is to restore power output during push-off. In the absence of such intervention, biofeedback that encourages increased push-off intensity shows promise to reverse the characteristic redistribution of joint power generation due to age (Lewis \& Ferris 2008; Browne \& Franz 2017a). Knowing the individual-muscle metabolic response to enhanced push-off intensity would allow scientists and 
clinicians to design better rehabilitation therapies for individualized prescription with translational potential to elicit more youthful and efficient gait biomechanics. Although our young adult population are not representative of those that would require such intervention, our data provide an important reference for future bioenergetic modeling studies in the target populations.

Our simulations reveal an increased metabolic cost of operating muscles spanning the hip, knee, and ankle when targeting larger than normal $\mathrm{F}_{\mathrm{P}}-$ a response that manifested most during single support. At the hip, this metabolic penalty is explained primarily by increases for the gluteus medius and maximus during early single support and the hamstrings during midstance. Those changes may arise from larger than normal braking forces during early stance and larger acceleration of the center of mass during midstance, respectively. We predict that these metabolic changes would be mitigated if walking speed was permitted to increase in response to larger propulsive forces. This methodological design may also explain why we found no increase in the metabolic cost of muscles spanning the ankle during trailing limb double support when targeted larger than normal $F_{P}$. Although this outcome is surprising, it is consistent with recent ultrasound imaging data alluding to a potentially counterproductive trade-off between increased activation and shorter operating lengths on force transmission from the calf muscles during push-off (Browne \& Franz In Revision).

In contrast to effects when targeting $40 \%$ larger $\mathrm{F}_{\mathrm{P}}$, and despite higher whole-body metabolic cost on average, targeting a more modest $20 \%$ increase in Fp resulted in lower metabolic costs to operate muscles spanning the hip and knee during leading limb double support. Here, the hamstring muscles appear to consume lower average metabolic cost during early stance compared to Norm. Thereafter, these reductions are overcome by increased costs during single support and leg swing. This suggests it may be theoretically possible to reduce metabolic costs of operating individual leg muscles to values lower than predicted for normal walking. Although at odds with generally accepted theories regarding energy optimization in walking (Browne \& Franz 2017b), such a conclusion at the individual muscle level may allude to patterns of inter-muscular coordination that are outside the scope of this study but warrant further investigation.

Compared to walking with smaller $\mathrm{F}_{\mathrm{P}}$, changes in the metabolic costs of operating individual leg muscles are much more evenly allocated when walking with larger FP. It is difficult to predict whether to expect the same outcome in older adults or people with gait pathology in response to interventions designed to restore F. However, our results suggest that such interventions should consider not only the metabolic consequences of local (e.g., ankle) assistance to increase $\mathrm{F}_{\mathrm{P}}$, but also any resultant compensation at other joints. Moving forward, we envision rehabilitative programs and assistive technologies that are informed by and objectively evaluated via individual muscle metabolic responses derived via the bioenergetic simulations used in this study.

\section{Limitations}

We first acknowledge other factors that contribute to increased metabolic cost of walking in older adults. These may include changes in trunk flexion (Carey \& Crompton 2005; Boyer et al. 2017), muscle biochemistry and mitochondria content (Brooks et al. 2005), and decreased tendon stiffness (Onambele et al. 2006). Our study instead used experimental manipulations in younger subjects to test for the metabolic consequences of biomechanical factors in the absence of other known age-related changes. We have reasonable evidence that the Fp biofeedback provides a method for young adults to emulate patterns of joint kinetics characteristic of older adults (DeVita \& Hortobagyi 2000; Boyer et al. 2017; Browne \& Franz 2017a; Browne \& Franz 2019). 
However, because our participants were not older adults, future work will need to test the sensitivity of these simulations to variations in model parameters. Another limitation we recognize is that we did not measure kinematics for the subtalar or metatarsal-phalangeal joints or the upper body. To overcome part of this limitation, we used trunk kinematics averaged from another cohort in our simulations. For transparency, our appendix documents these methodological considerations in more detail. Finally, participants walked at a fixed speed despite biofeedback-induced changes in F. This led to differences in stride frequency known to affect metabolic cost (Donelan et al. 2002; Kuo et al. 2005; Soo \& Donelan 2010) and likely to confound our results in ways described earlier.

\section{Conclusion}

This study provides individual muscle-level insight into the metabolic consequences of walking with less and more vigorous push-off during walking. We first conclude that walking with insufficient push-off intensity places higher mechanical demand on muscles spanning the hip which in turn increases the metabolic cost of walking. This suggests that a distal-to-proximal redistribution of muscle demand during walking may independently contribute to higher metabolic costs in older adults and those with gait pathology. We also conclude that a fundamentally different and more evenly-allocated pattern of increased muscular demand explains higher metabolic energy costs when targeting larger propulsive forces. More generally, our simulations provide an initial benchmark for the metabolic consequences of altered propulsive force generation during walking that can inform strategies to improve walking economy in those with deficits in push-off intensity. Finding ways to preserve or restore ankle power generation, via rehabilitation, assistive technologies, or other means, appears central not only to improve walking performance, but also to improve walking economy.

\section{Acknowledgements}

This study was supported by grants from the National Institutes of Health (R01AG058615) and Parkinson's Foundation (PF-VSA-SFW-1908). We thank our participants for donating their time to participate in this study. We also thank Emily McCain, Rebecca Krupenevich, Gabriella Diaz, and Sidney Baudendistel for their expertise and assistance in study design, data collection, data processing, and modeling procedures. 


\section{References}

1. Arnold EM, Hamner SR, Seth A, Millard M, Delp SL. 2013. How muscle fiber lengths and velocities affect muscle force generation as humans walk and run at different speeds. J Exp Biol. 216(11):2150-2160.

2. Bell AL, Pedersen DR, Brand RA. 1990. A Comparison of the Accuracy of Several Hip Center. J Biomech. 23(November):6-8.

3. Bhargava LJ, Pandy MG, Anderson FC. 2004. A phenomenological model for estimating metabolic energy consumption in muscle contraction. J Biomech. 37(1):81-88.

4. Boyer KA, Johnson RT, Banks JJ, Jewell C, Hafer JF. 2017. Systematic review and metaanalysis of gait mechanics in young and older adults. Exp Gerontol. 95(September):63-70.

5. Brockway JM. 1987. Brockway_1987_HumNut - Derivation of formulae used to calculate energy expenditure in man.pdf. Hum Nutr Clin Nutr. 41(C):463-71.

6. Brooks GA, Fahey TD, Baldwin KM. 2005. Exercise Physiology: Human Bioenergetics and Its Applications. Fourth Edi. [place unknown]: McGraw Hill.

7. Browne MG, Franz JR. Increasing plantarflexor activity during walking may elicit counterproductive effects on fascicle behavior in older adults. Gait Posture.

8. Browne MG, Franz JR. 2017a. The independent effects of speed and propulsive force on joint power generation in walking. J Biomech. 55:48-55.

9. Browne MG, Franz JR. 2017b. Does dynamic stability govern propulsive force generation in human walking? R Soc Open Sci. 4(11).

10. Browne MG, Franz JR. 2019. Ankle power biofeedback attenuates the distal-to-proximal redistribution in older adults. Gait Posture. 71(February):44-49.

11. Cappozzo A. 1984. Gait analysis methodology. Hum Mov Sci. 3(1-2):27-50.

12. Carey TS, Crompton RH. 2005. The metabolic costs of "bent-hip, bent-knee" walking in humans. J Hum Evol. 48(1):25-44.

13. Cofré LE, Lythgo N, Morgan D, Galea MP. 2011. Aging modifies joint power and work when gait speeds are matched. Gait Posture. 33(3):484-489.

14. Delp SL, Anderson FC, Arnold AS, Loan P, Habib A, John CT, Guendelman E, Thelen DG. 2007. OpenSim: Open-source software to create and analyze dynamic simulations of movement. IEEE Trans Biomed Eng. 54(11):1940-1950.

15. DeVita P, Hortobagyi T. 2000. Age causes a redistribution of joint torques and powers during gait. J Appl Physiol. 88(5):1804-1811.

16. Donelan JM, Kram R, Kuo AD. 2002. Mechanical work for step-to-step transitions is a major determinant of the metabolic cost of human walking. J Exp Biol. 205(23):3717-3727.

17. Fickey SN, Browne MG, Franz JR. 2018. Biomechanical effects of augmented ankle power output during human walking. J Exp Biol. 221(22).

18. Franz JR. 2016. The Age-Associated Reduction in Propulsive Power Generation in Walking. Exerc Sport Sci Rev. 44(4):129-136.

19. Handsfield GG, Meyer CH, Hart JM, Abel MF, Blemker SS. 2014. Relationships of 35 lower limb muscles to height and body mass quantified using MRI. J Biomech. 47(3):631-638.

20. Hardy SE, Perera S, Roumani YF, Chandler JM, Studenski SA. 2007. Improvement in usual gait speed predicts better survival in older adults. J Am Geriatr Soc. 55(11):1727-1734.

21. Hicks JL, Uchida TK, Seth A, Rajagopal A, Delp SL. 2015. Is My Model Good Enough? Best Practices for Verification and Validation of Musculoskeletal Models and Simulations of Movement. J Biomech Eng. 137(2).

22. Hortobágyi T, Finch A, Solnik S, Rider P, De Vita P. 2011. Association between muscle 
activation and metabolic cost of walking in young and old adults. Journals Gerontol - Ser A Biol Sci Med Sci. 66 A(5):541-547.

23. Huang TWP, Shorter KA, Adamczyk PG, Kuo AD. 2015. Mechanical and energetic consequences of reduced ankle plantar-flexion in human walking. J Exp Biol. 218(22):35413550 .

24. Jee L, Rasmussen H, Caspi A, Ambler A, Broadbent JM, Cohen HJ, Arbeloff T, Rasmussen LJH, Caspi A, Ambler A, et al. 2019. Association of Neurocognitive and Physical Function With Gait Speed in Midlife. JAMA Netw Open. 2(10):1-15.

25. Judge JO, Davis RB, Ounpuu S. 1996. Step Length Reductions in Advanced Age: The Role of Ankle and Hip Kinetics. Journals Gerontol Ser A Biol Sci Med Sci. 51A(6):M303-M312.

26. Kerrigan DC, Todd MK, Della Croce U, Lipsitz LA, Collins JJ. 1998. Biomechanical gait alterations independent of speed in the healthy elderly: Evidence for specific limiting impairments. Arch Phys Med Rehabil. 79(3):317-322.

27. Kikkert LHJ, Vuillerme N, van Campen JP, Hortobágyi T, Lamoth CJ. 2016. Walking ability to predict future cognitive decline in old adults: A scoping review. Ageing Res Rev. 27:1-14.

28. Kim M, Collins SH. 2015. Once-per-step control of ankle-foot prosthesis push-off work reduces effort associated with balance during walking. J Neuroeng Rehabil. 12(43).

29. Koelewijn AD, Heinrich D, van den Bogert AJ. 2019. Metabolic cost calculations of gait using musculoskeletal energy models, a comparison study.Grabowski A, editor. PLoS One. 14(9):e0222037.

30. Kuo AD, Donelan JM, Ruina A. 2005. Energetic consequences of walking like an inverted pendulum: Step-to-step transitions. Exerc Sport Sci Rev. 33(2):88-97.

31. Lewis CL, Ferris DP. 2008. Walking with increased ankle pushoff decreases hip muscle moments. J Biomech. 41(10):2082-2089.

32. Mian OS, Thom JM, Ardigò LP, Morse CI, Narici M V., Minetti AE. 2007. Effect of a 12month physical conditioning programme on the metabolic cost of walking in healthy older adults. Eur J Appl Physiol. 100(5):499-505.

33. Miller RH. 2014. A comparison of muscle energy models for simulating human walking in three dimensions. J Biomech. 47(6):1373-1381.

34. Morris JN, Hardman AE. 1997. Walking to health. Sport Med. 23(5):306-332.

35. Onambele GL, Narici M V., Maganaris CN. 2006. Calf muscle-tendon properties and postural balance in old age. J Appl Physiol. 100(6):2048-2056.

36. Orselli MI V., Franz JR, Thelen DG. 2017. The effects of Achilles tendon compliance on triceps surae mechanics and energetics in walking. J Biomech. 60:227-231.

37. Ortega JD, Farley CT. 2007. Individual limb work does not explain the greater metabolic cost of walking in elderly adults. J Appl Physiol. 102(6):2266-2273.

38. Ortega JD, Fehlman LA, Farley CT. 2008. Effects of aging and arm swing on the metabolic cost of stability in human walking. J Biomech. 41:3303-3308.

39. Pataky TC. 2012. One-dimensional statistical parametric mapping in Python. Comput Methods Biomech Biomed Engin. 15(3):295-301.

40. Peterson DS, Martin PE. 2010. Effects of age and walking speed on coactivation and cost of walking in healthy adults. Gait Posture. 31(3):355-359.

41. Piazza SJ, Okita N, Cavanagh PR. 2001. Accuracy of the functional method of hip joint center location: Effects of limited motion and varied implementation. J Biomech. 34(7):967-973.

42. Pieper NL, Baudendistel ST, Hass CJ, Diaz GB, Krupenevich RL, Franz JR. The metabolic and mechanical consequences of altered propulsive force generation in walking. $\mathrm{J}$ Biomech. 
43. Rajagopal A, Dembia CL, DeMers MS, Delp DD, Hicks JL, Delp SL. 2016. Full-Body Musculoskeletal Model for Muscle-Driven Simulation of Human Gait. IEEE Trans Biomed Eng. 63(10):2068-2079.

44. Reimann H, Fettrow T, Jeka JJ. 2018. Strategies for the Control of Balance During Locomotion.

45. Sawicki GS, Lewis CL, Ferris DP. 2009. It pays to have a spring in your step. Exerc Sport Sci Rev. 37(3):130-138.

46. Seth A, Hicks JL, Uchida TK, Habib A, Dembia CL, Dunne JJ, Ong CF, DeMers MS, Rajagopal A, Millard M, et al. 2018. OpenSim: Simulating musculoskeletal dynamics and neuromuscular control to study human and animal movement. PLoS Comput Biol. 14(7):e1006223.

47. Silder A, Heiderscheit B, Thelen DG. 2008. Active and passive contributions to joint kinetics during walking in older adults. J Biomech. 41(7):1520-1527.

48. Soo CH, Donelan JM. 2010. Mechanics and energetics of step-to-step transitions isolated from human walking. J Exp Biol. 213(24):4265-4271.

49. Uchida TK, Hicks JL, Dembia CL, Delp SL. 2016. Stretching Your Energetic Budget: How Tendon Compliance Affects the Metabolic Cost of Running.

50. Umberger BR. 2010. Stance and swing phase costs in human walking. J R Soc Interface. 7(50):1329-1340.

51. Umberger BR, Gerritsen KGM, Martin PE. 2003. A model of human muscle energy expenditure. Comput Methods Biomech Biomed Engin. 6(2):99-111.

52. World Health Organization. 2019. The top 10 causes of death, 2018. Fact Sheets [Internet].(November 24):1-9. https://www.who.int/news-room/fact-sheets/detail/the-top-10causes-of-death 


\section{Tables}

Table 1: Statistics for repeated measures ANOVA and LSD post-hoc pairwise tests corresponding to Figure 3. Conditions with significant p-values are bolded. We do not report pairwise differences for joint-level metabolic costs with insignificant ANOVA main effect (rows with missing data). $\eta_{\mathrm{p}}^{2}$ : partial eta squared, $d$ : Cohen's D, $p$ : pairwise p-value, Diff: mean difference versus Norm.

\begin{tabular}{|c|c|c|c|c|c|c|c|c|c|c|c|c|c|c|}
\hline \multirow{3}{*}{ Muscles } & \multirow{2}{*}{\multicolumn{2}{|c|}{$\begin{array}{l}\text { ANOVA Main } \\
\text { Effect }\end{array}$}} & \multicolumn{12}{|c|}{ Pairwise condition vs Norm } \\
\hline & & & \multicolumn{3}{|c|}{$-40 \%$} & \multicolumn{3}{|c|}{$-20 \%$} & \multicolumn{3}{|c|}{$+20 \%$} & \multicolumn{3}{|c|}{$+40 \%$} \\
\hline & $p$ & $\eta_{p}^{2}$ & $\begin{array}{l}\text { Diff } \\
(W / k g)\end{array}$ & $p$ & $d$ & $\begin{array}{c}\text { Diff } \\
(W / k g)\end{array}$ & $p$ & $d$ & $\begin{array}{c}\text { Diff } \\
(W / k g)\end{array}$ & $p$ & $d$ & $\begin{array}{c}\text { Diff } \\
(W / k g)\end{array}$ & $p$ & $d$ \\
\hline \multicolumn{15}{|c|}{ Entire Stride } \\
\hline All & 0.010 & 0.040 & +0.45 & 0.026 & 0.549 & +0.06 & 0.505 & 0.075 & +0.18 & 0.105 & 0.214 & +0.42 & 0.003 & 0.517 \\
\hline Hip & 0.002 & 0.059 & +0.29 & 0.008 & 0.713 & +0.06 & 0.291 & 0.146 & +0.04 & 0.240 & 0.097 & +0.20 & 0.008 & 0.492 \\
\hline Ankle & 0.035 & 0.065 & -0.05 & 0.370 & -0.223 & -0.02 & 0.735 & -0.075 & +0.11 & 0.089 & 0.538 & +0.10 & 0.035 & 0.488 \\
\hline \multicolumn{15}{|c|}{$\begin{array}{l}\text { Leading Limb Double } \\
\text { Support }\end{array}$} \\
\hline All & $<0.001$ & 0.239 & +0.17 & 0.036 & 1.249 & +0.12 & 0.064 & 0.896 & -0.14 & $<0.001$ & -1.027 & -0.07 & 0.097 & -0.529 \\
\hline Hip & $<0.001$ & 0.304 & +0.16 & 0.005 & 2.495 & +0.10 & 0.014 & 1.535 & -0.05 & 0.001 & -0.842 & +0.00 & 0.843 & 0.070 \\
\hline All & $<0.001$ & 0.181 & +0.19 & 0.068 & 0.931 & -0.03 & 0.744 & -0.127 & +0.20 & $<0.001$ & 0.954 & +0.37 & $<0.001$ & 1.788 \\
\hline Hip & 0.002 & 0.099 & +0.04 & 0.378 & 0.350 & -0.03 & 0.407 & -0.248 & +0.05 & $<0.001$ & 0.431 & +0.13 & 0.003 & 1.044 \\
\hline Knee & $<0.001$ & 0.184 & +0.06 & 0.053 & 1.093 & -0.01 & 0.804 & -0.103 & +0.06 & 0.004 & 1.105 & +0.13 & $<0.001$ & 2.284 \\
\hline Ankle & 0.003 & 0.186 & +0.07 & 0.049 & 1.418 & +0.00 & 0.971 & 0.017 & +0.06 & $<0.001$ & 1.086 & +0.09 & $<0.001$ & 1.808 \\
\hline \multicolumn{15}{|c|}{$\begin{array}{l}\text { Trailing Limb Double } \\
\text { Support }\end{array}$} \\
\hline All & 0.541 & 0.025 & - & - & - & - & - & - & - & - & - & - & - & - \\
\hline Hip & 0.092 & 0.051 & - & - & - & - & - & - & - & - & - & - & - & - \\
\hline Knee & 0.927 & 0.007 & - & - & - & - & - & - & - & - & - & - & - & - \\
\hline \multicolumn{15}{|l|}{ Swing } \\
\hline
\end{tabular}


Table 2: Gait cycle periods of interest for each biofeedback condition in absolute (s) and relative (\%) time. These periods were calculated over 10 strides in the window of best biofeedback targeting performance (i.e., best matching the $-40 \%$, $-20 \%$, etc. of $\mathrm{F}_{\mathrm{P}}$ ). Bolded values indicate significant post-hoc pairwise difference versus Norm. These are the periods over which the jointlevel metabolic costs were integrated in Figure 3. Stride time is defined as $100 \%$ of the gait cycle, thus we omit these data and run post hoc analyses using duration. SD: standard deviation across participant means, s: seconds, \%: percent of gait cycle

\begin{tabular}{|c|c|c|c|c|c|c|c|c|c|c|c|c|}
\hline \multirow{2}{*}{\multicolumn{2}{|c|}{$\begin{array}{l}\text { Biofeedback Condition } \\
\text { Units }\end{array}$}} & \multicolumn{2}{|c|}{-40} & \multicolumn{2}{|c|}{-20} & \multicolumn{2}{|c|}{ Norm } & \multicolumn{2}{|c|}{+20} & \multicolumn{2}{|c|}{+40} & \multirow{2}{*}{$\begin{array}{c}\text { ANOVA } \\
p\end{array}$} \\
\hline & & $s$ & $\%$ & $s$ & $\%$ & $s$ & $\%$ & $s$ & $\%$ & $s$ & $\%$ & \\
\hline \multirow{2}{*}{$\begin{array}{l}\text { Gait Cycle } \\
\text { Duration }\end{array}$} & Mean & 0.79 & - & 0.89 & - & 1.05 & - & 1.07 & - & 1.15 & - & \multirow{2}{*}{$<0.001$} \\
\hline & $S D$ & 0.10 & - & 0.08 & - & 0.11 & - & 0.09 & - & 0.13 & - & \\
\hline \multirow{2}{*}{$\begin{array}{l}\text { Double Support } \\
\text { Leading Limb }\end{array}$} & Mean & 0.11 & 14.77 & 0.14 & 16.05 & 0.16 & 15.35 & 0.15 & 14.02 & 0.16 & 13.69 & \multirow{2}{*}{$<0.001$} \\
\hline & $S D$ & 0.04 & 4.96 & 0.02 & 1.97 & 0.02 & 1.31 & 0.02 & 1.24 & 0.02 & 1.22 & \\
\hline \multirow{2}{*}{ Single Support } & Mean & 0.26 & 33.57 & 0.30 & 33.89 & 0.36 & 34.23 & 0.39 & 35.96 & 0.42 & 36.32 & \multirow{2}{*}{$<0.001$} \\
\hline & $S D$ & 0.04 & 2.25 & 0.03 & 2.00 & 0.02 & 2.00 & 0.04 & 1.19 & 0.05 & 1.25 & \\
\hline \multirow{2}{*}{$\begin{array}{l}\text { Double Support } \\
\text { Trailing Limb }\end{array}$} & Mean & 0.15 & 18.22 & 0.14 & 16.19 & 0.17 & 16.13 & 0.15 & 14.10 & 0.16 & 13.75 & \multirow{2}{*}{0.488} \\
\hline & $S D$ & 0.08 & 8.25 & 0.02 & 2.03 & 0.05 & 3.14 & 0.02 & 1.28 & 0.02 & 1.26 & \\
\hline \multirow{2}{*}{ Swing } & Mean & 0.26 & 33.44 & 0.30 & 33.87 & 0.36 & 34.29 & 0.39 & 35.93 & 0.42 & 36.24 & \multirow{2}{*}{$<0.001$} \\
\hline & $S D$ & 0.04 & 2.87 & 0.03 & 1.99 & 0.02 & 1.97 & 0.04 & 1.33 & 0.05 & 1.24 & \\
\hline
\end{tabular}




\section{Figure Captions}

Figure 1: Participants pushed off the ground with varying vigor to target $-40 \%,-20 \%,+20 \%$, $+40 \%$, and their typical (Norm) propulsive force $\left(\mathrm{F}_{\mathrm{P}}\right)$ in a walking biofeedback paradigm with 5minute trials per condition. Participant anthropometrics, marker trajectories, and ground reaction forces from the final 2 minutes of each trial defined musculoskeletal modeling simulations. We simulated the first left and right full stride from the 10 second window with the most accurate biofeedback targeting performance. From these simulations, we ultimately determined the metabolic power required from each lower body muscle to drive the scaled model and match the measured movements.

Figure 2: For each biofeedback condition, we display the walking metabolic power empirically measured using indirect calorimetry (A) and simulated from two different musculoskeletal metabolic models (B, C). The models produced nearly identical results (D) that correlated moderately with empirically-measured metabolic power $(\mathrm{E}, \mathrm{F})$, explaining about $1 / 3^{\text {rd }}$ of the variance. Musculoskeletal modeling of metabolic power allows researchers to estimate the metabolic power required for lower body muscles to drive a simulation of each participant's individual walking pattern. This allows for comparison across the FP biofeedback conditions at specific instances of the gait cycle $(\mathrm{G})$. Given the high similarity of the two models, we opted to use the Umberger model for our metabolic cost estimates and statistical comparisons.

Figure 3: For each biofeedback condition, we show net metabolic power summed for all modeled lower body muscles (A), and for those spanning each of the major joints of the lower limb (B, C, D). Metabolic powers were integrated over: the entire stride, leading limb double support, single limb support, trailing limb double support, and swing phase. Asterisks show significant LSD post hoc pairwise differences versus Norm in the presence of a significant ANOVA main effect. On the right, we also display instantaneous net metabolic power across the gait cycle.

Figure 4: We plot the metabolic powers across the gait cycle for the 21 highest power-consuming muscles of the lower limb for each biofeedback condition. Gray shaded regions show periods with a significant repeated measures ANOVA main effect for biofeedback condition using statistical parametric mapping. Colored blocks identify any LSD post hoc pairwise differences for that respective colored condition versus Norm. OpenSim muscles with multiple lines of action (gluteus minimus/medius/maximus and adductor magnus) were summed for simplicity. 


\section{Appendix}

\section{Model Efficacy}

For the first 5 supplementary figures we show simulation results for all subjects, across all conditions, and on both sides for transparent reporting of simulation environments. In the final two supplementary figures we show results by condition averaged bilaterally and across participants.

In Supplementary Figure S1 we show joint kinematics which varied consistently across subjects, mainly occurring in the sagittal plane. In Supplementary Figure S2, we display the "hand of god" global forces and moments required to support each model in its environment while walking. The horizontal dashed black lines show the thresholds commonly recommended as optimal for a given trial ( $25 \mathrm{~N}$ forces \& $75 \mathrm{Nm}$ moments per OpenSim documentation). Some measurable deviations were present, tended to occur during double support, and were mostly concentrated in the vertical forces and sagittal plane moments. Joint moment reserves (i.e., those not accounted for by modeled muscle-tendon units) in Supplementary Figure S3 had fewer outliers than the global forces and moments, but a few persisted especially regarding hip flexion, hip adduction, hip rotation, and knee flexion.

Subject 10 was our primary outlier for reserve moments and forces (light orange), which we note carried through to the muscle forces (Supplementary Figure S4) and activation levels (Supplementary Figure S5). Specifically, we note that the hamstrings and gracillis muscle outcomes differed from other simulations between $40 \%$ and $60 \%$ of the gait cycle. We processed other strides for this participant and condition, and all yielded a similar response. We note that this one outlier occurred in the 40\% larger FP condition and may disproportionately contribute to the elevated metabolic cost of the biarticular hamstring muscles shown in Figure 4. Reassuringly, although the average profile for that condition was likely affected by this outlier, our SPM analysis correctly identified this region to have no condition main effect.

Moving on, muscle forces, normalized to body mass, were stable and, other than for Subject 10 as described, were fairly similar across all subjects (Supplementary Figure S4). Computed muscle activation levels (Supplementary Figure S5) were mostly uniform and temporally similar across all subjects. Though, Subject 2 tended to consistently activate muscles at a higher level than most other subjects. When averaging for each condition, the simulated muscle forces (Supplementary Figure S6) and activation levels (Supplementary Figure S7) displayed many features that align with the smaller and larger $\mathrm{F}_{\mathrm{P}}$, similar to the metabolic cost profiles presented in our primary document.

\section{Addressing Model Efficacy}

We understand that some of our simulations do not follow best practice recommendations (Hicks et al. 2015). The virtual trunk kinematics are likely the primary source of these discrepancies. The only other viable option to address the lack of torso kinematics was to place the full mass of the torso within the pelvis segment. However, we were concerned that this would affect the model center of mass to a higher degree than a virtual torso segment. Thus, we pressed on with the virtual torso kinematics superimposed on each participant's lower body kinematics.

Another potential source of error may arise from scaling anatomical markers between the original model and their locations that depend on each participant's anthropometrics. During the scaling process, we identified the average anatomical marker root mean square error of $0.016 \pm 0.004 \mathrm{~m}$ (mean \pm standard deviation), with a max anatomical marker root mean square error of $0.0459 \mathrm{~m}$ (Subject 3, left posterior superior iliac spine). We also note that subjects with higher BMIs tended to have higher scale error. Many of the anatomical marker locations help define limb 
segment dimensions and coordinate axes, thus errors during the model scaling process may carry forward into future model estimates.

Even with these limitations, we were able to demonstrate typical ranges of kinematics (even for the trunk), realistic muscle activation levels, and stable muscle forces across the gait cycle. To mitigate the effect of any simulation outliers, we averaged each pair of left and rightsided trials together when analyzing our primary outcome measure - muscle metabolic power. Finally, when reporting our metabolic costs, we present the individual data points for each gait cycle period of interest so that our readers may consider the results on an individual-subject level alongside the averages or boxplots for the group or condition. We believe this analysis can make a significant contribution to the field to understand individual muscle metabolic costs while walking across a range of $\mathrm{F}_{\mathrm{P}}$, however we suggest that future investigations address the limitations we mention above.

\section{Supplemental Figure Captions}

Supplemental Figure S1: Joint kinematics across the gait cycle for all conditions in each participant.

Supplemental Figure S2: Global forces and moments across the gait cycle for all conditions in each participant.

Supplemental Figure S3: Net joint moment reserves moments across the gait cycle for all conditions in each participant.

Supplemental Figure S4: Computed muscle activation levels across the gait cycle for all conditions in each participant.

Supplemental Figure S5: Relative simulated activation levels across the gait cycle for all conditions in each participant.

Supplemental Figure S6: Condition average muscle forces for each of the 21 highest powerconsuming muscles of the lower limb.

Supplemental Figure S7: Condition average muscle activation levels for each of the 21 highest power-consuming muscles of the lower limb. 
Figure 2
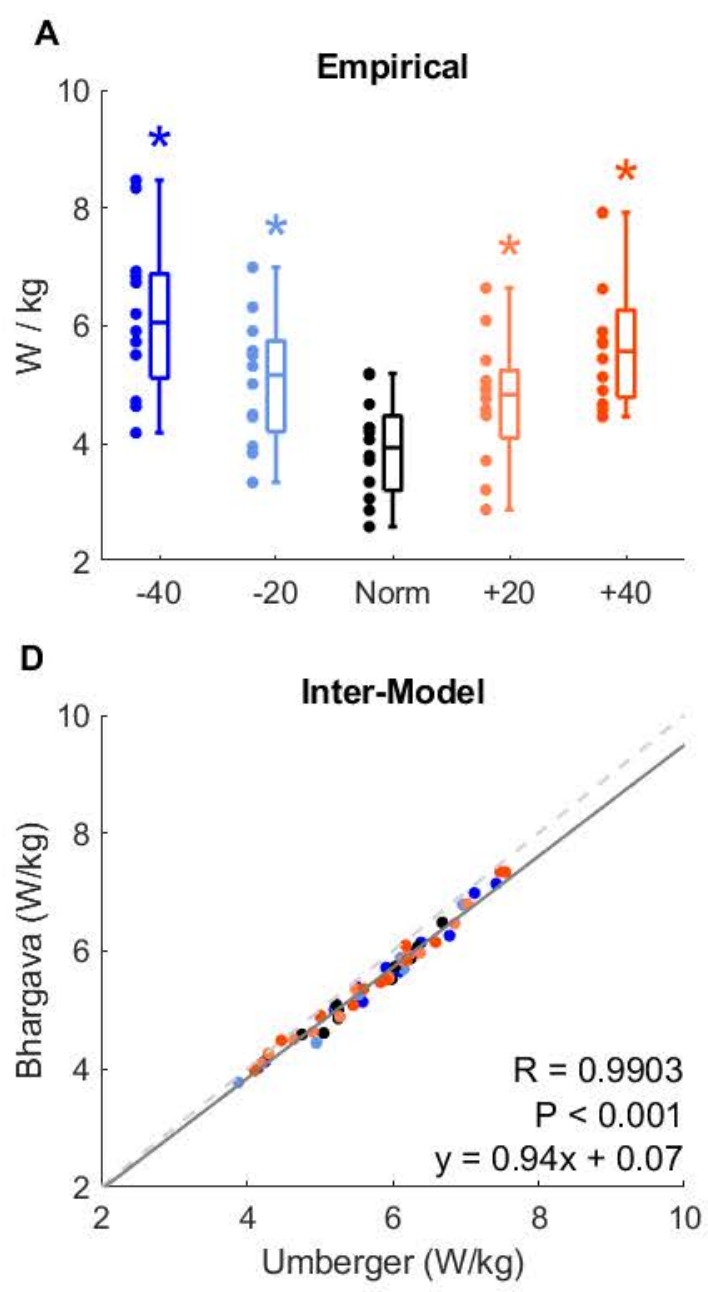

G

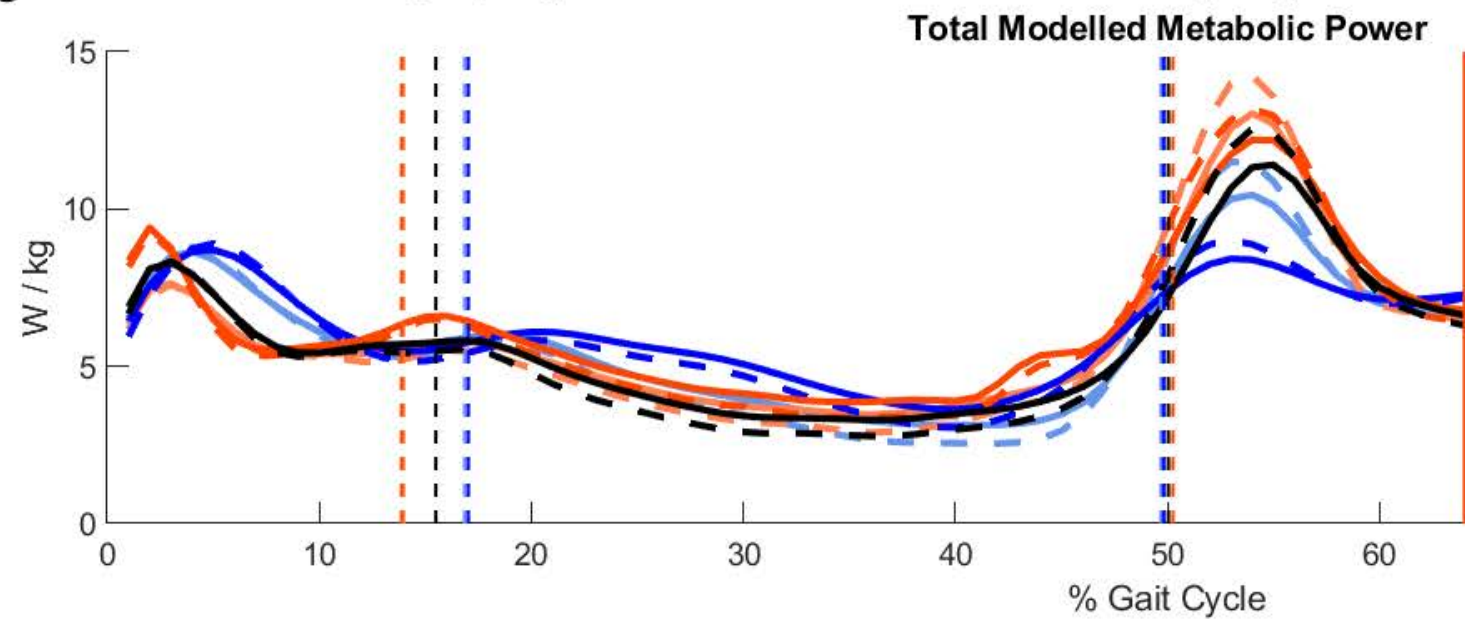

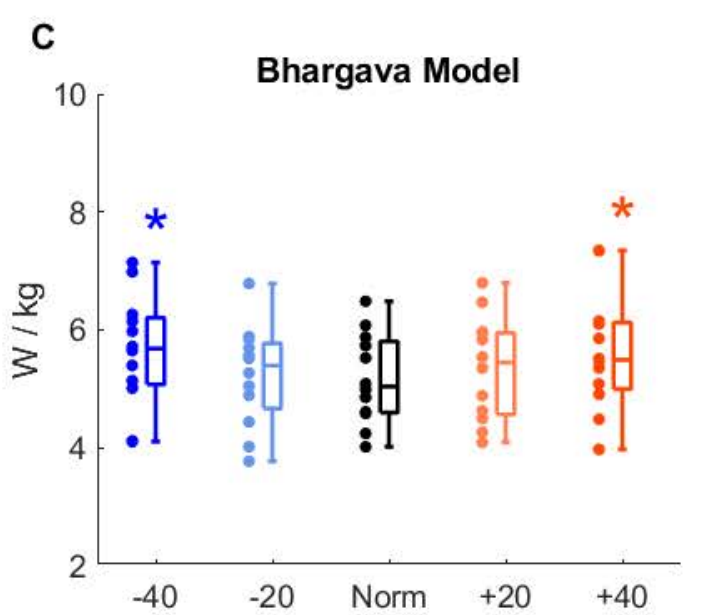

E
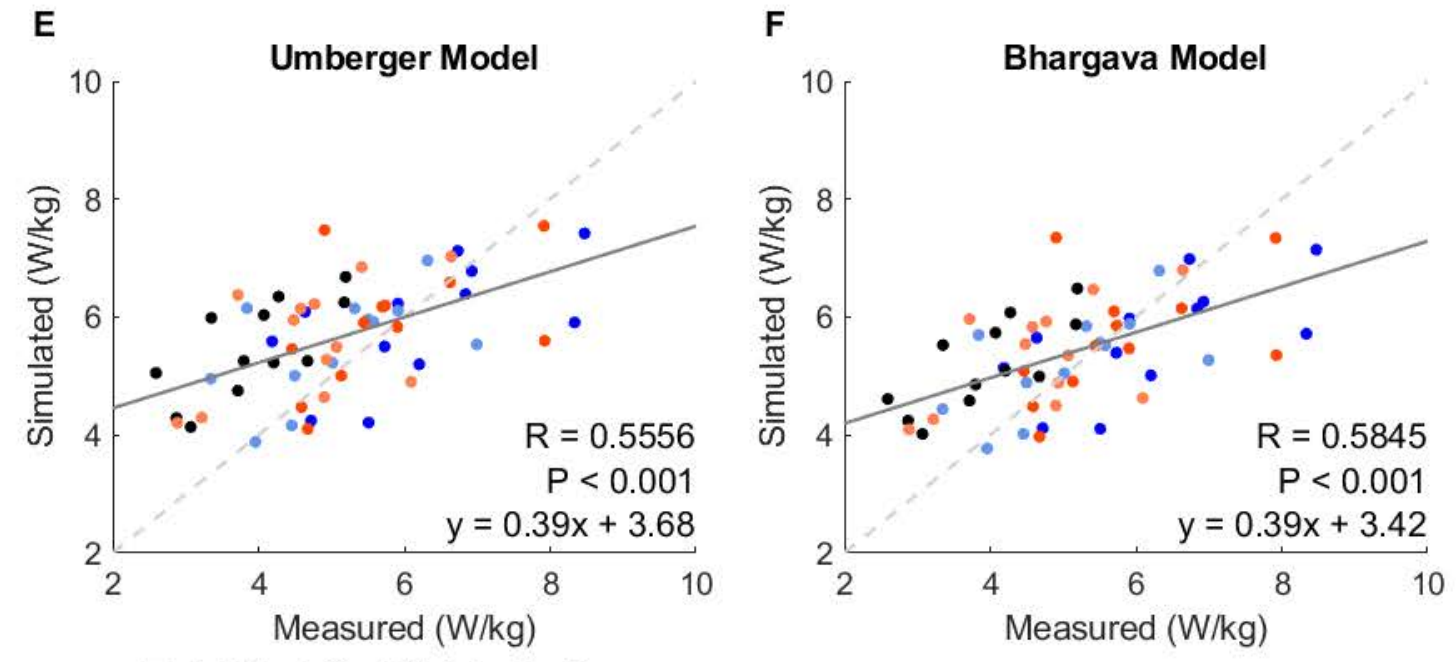

- Umberger

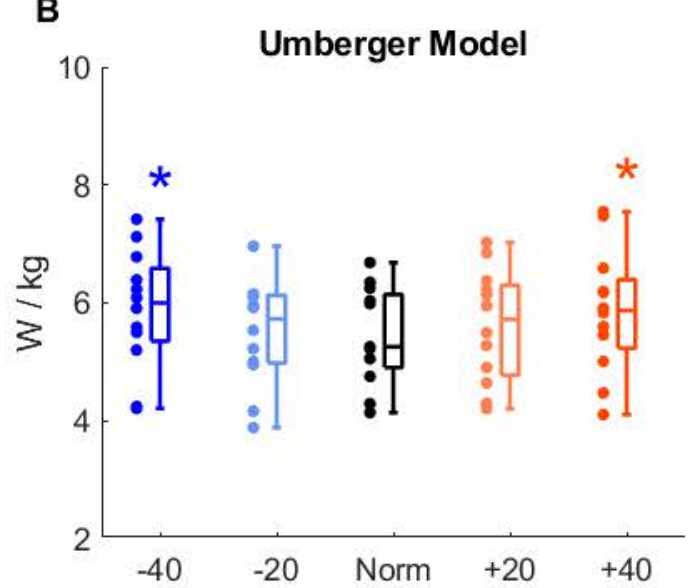

F

- - Bhargava 
Trailing Limb Double Support
Figure 3

A

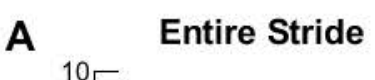

Leading Limb

Double Support ALL MUSCLES

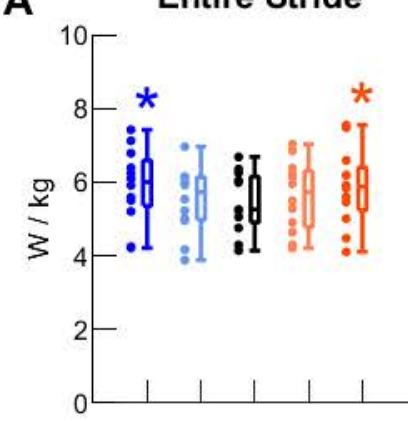

Single Support

Swing Phase

Gait Cycle

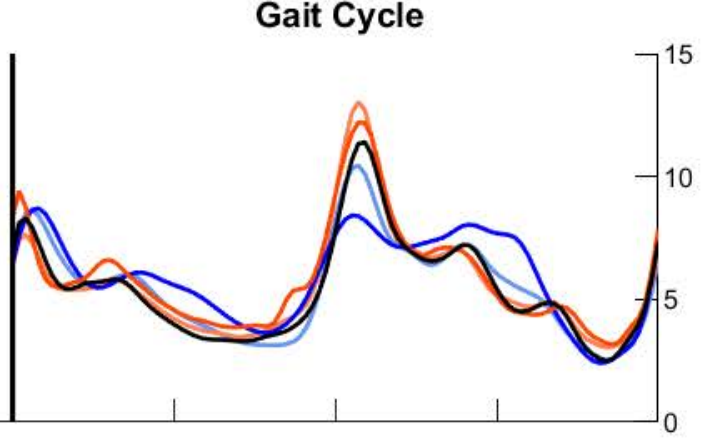

*

B
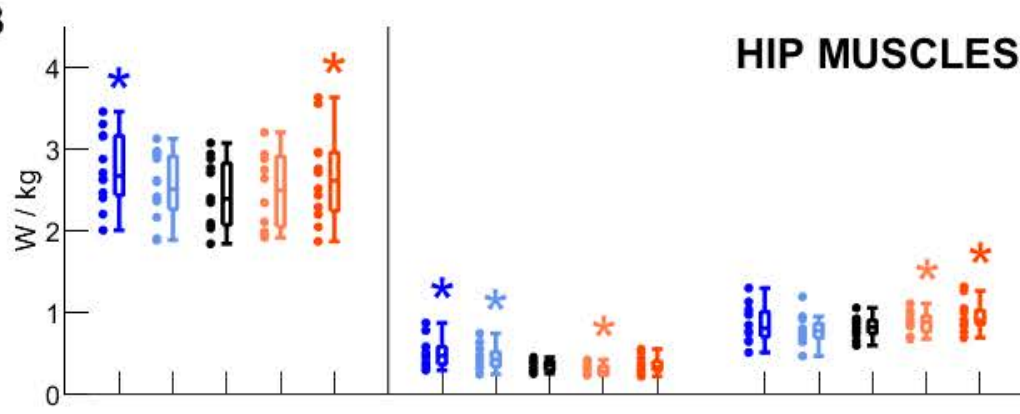

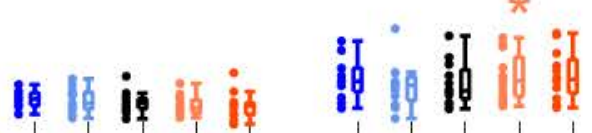

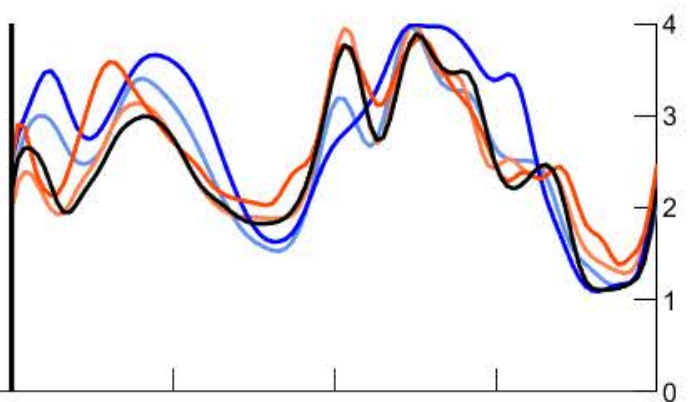

C

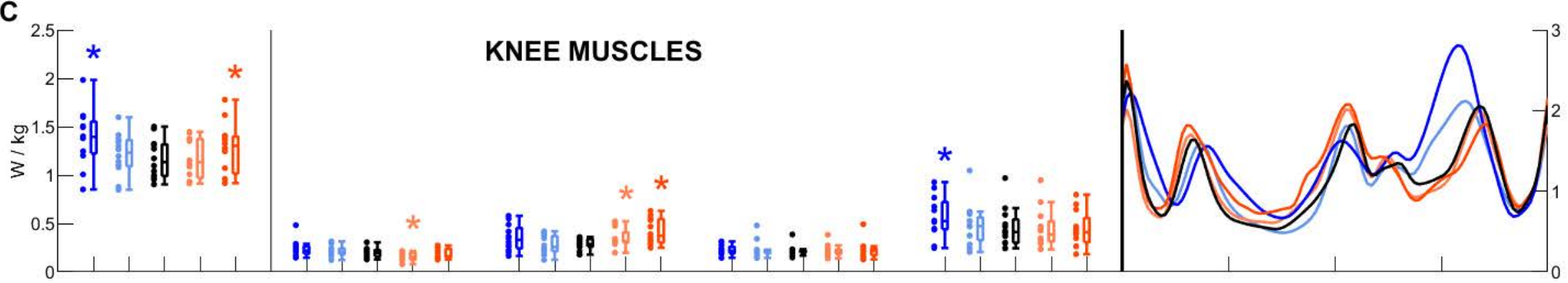

D

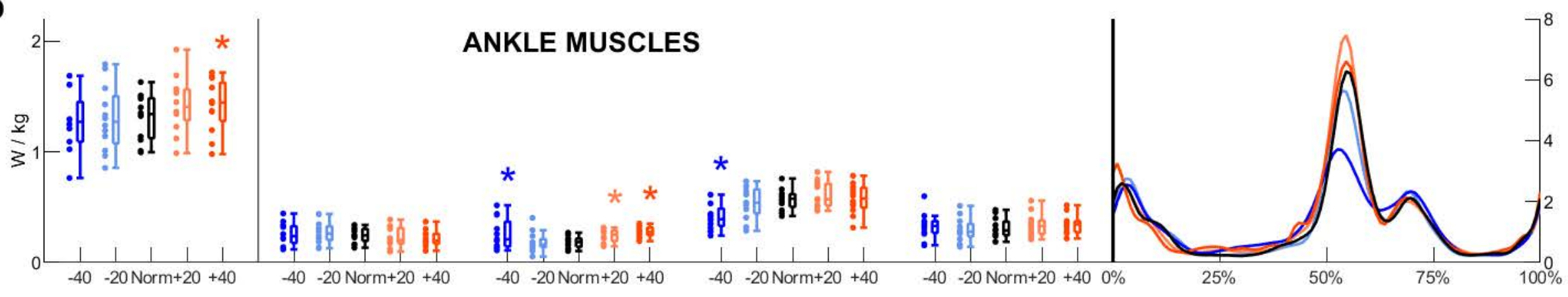



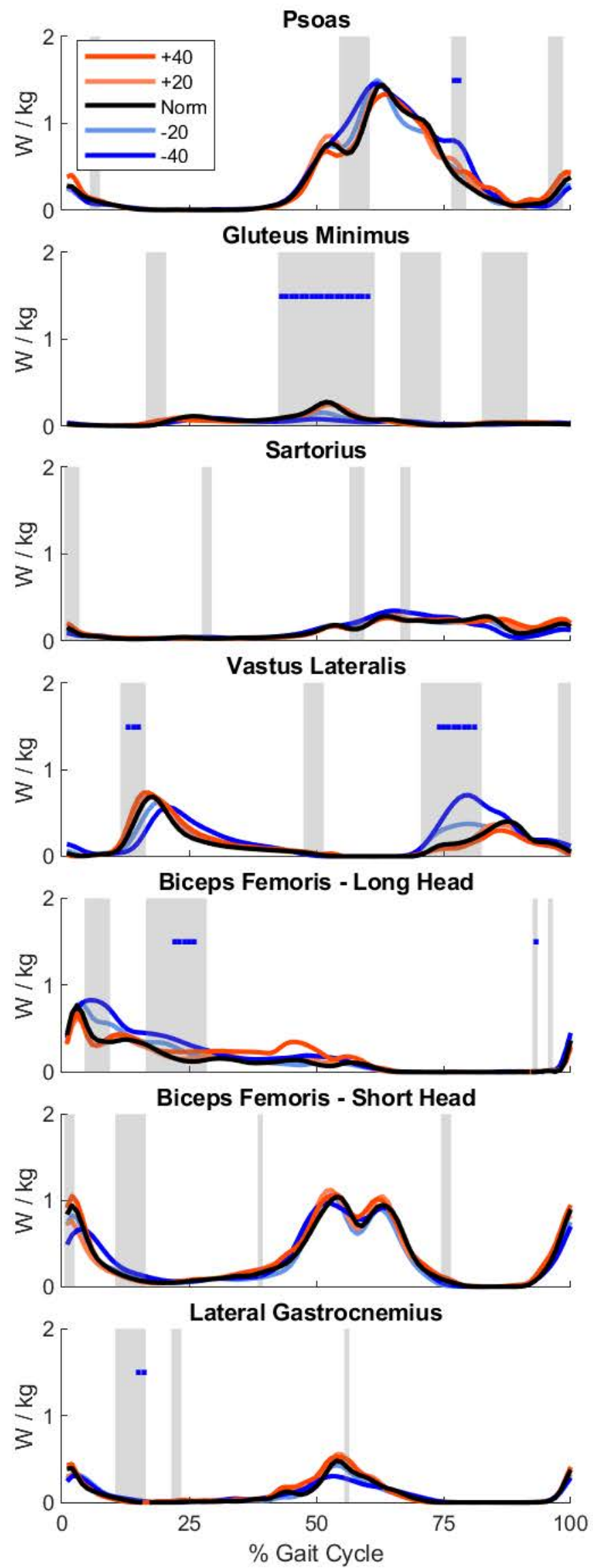
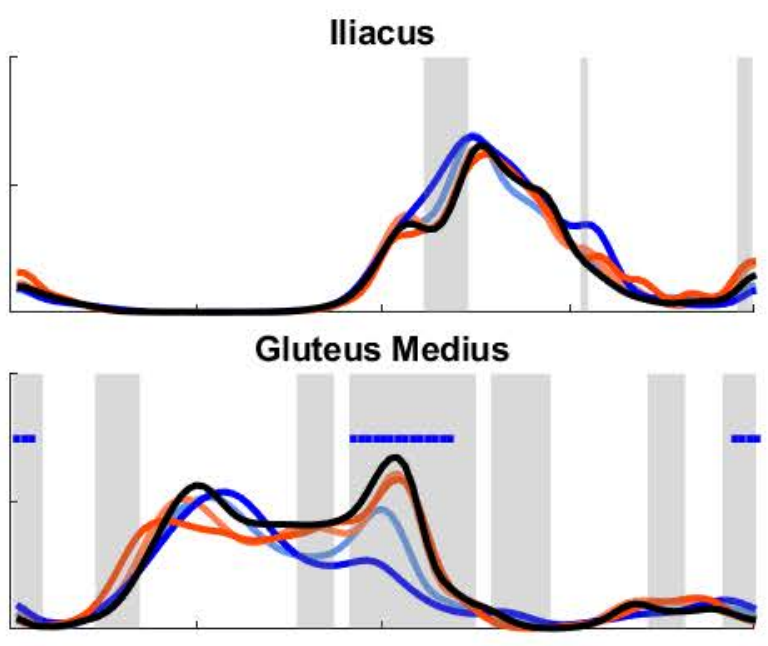

Adductor Magnus

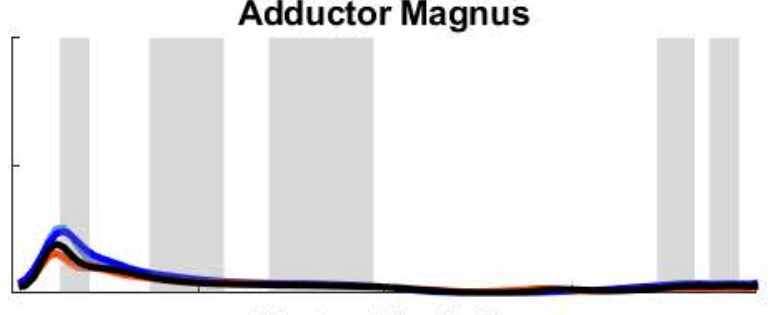

Vastus Medialis

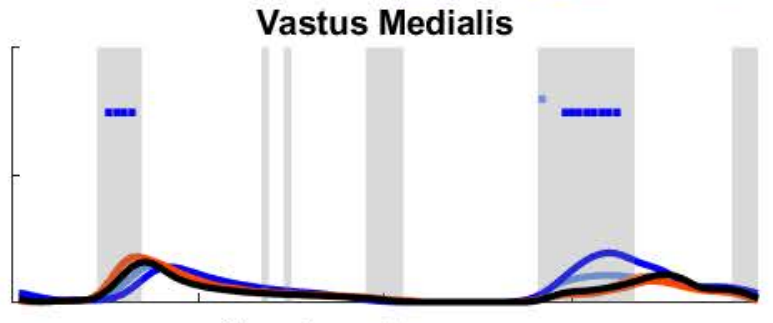

Semimembranosus

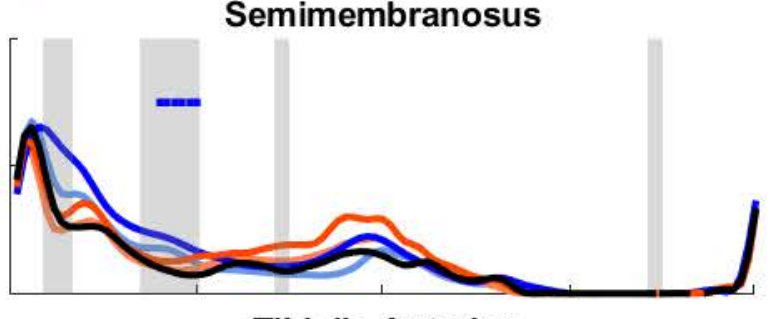

Tibialis Anterior

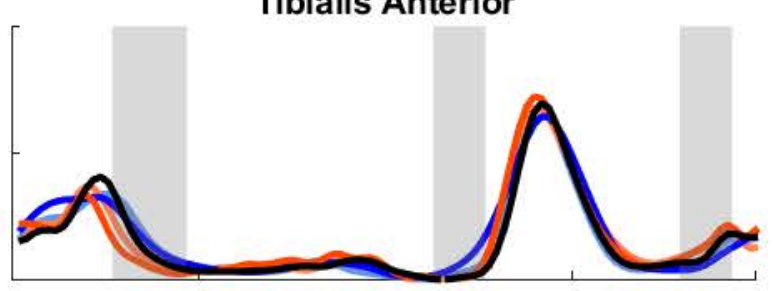

Medial Gastrocnemius

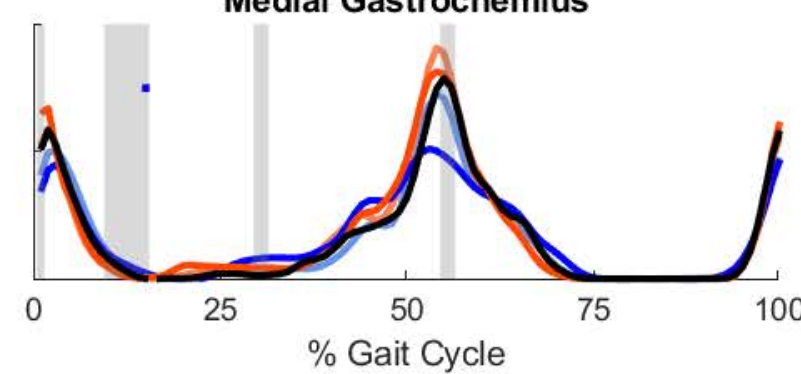

Rectus Femoris
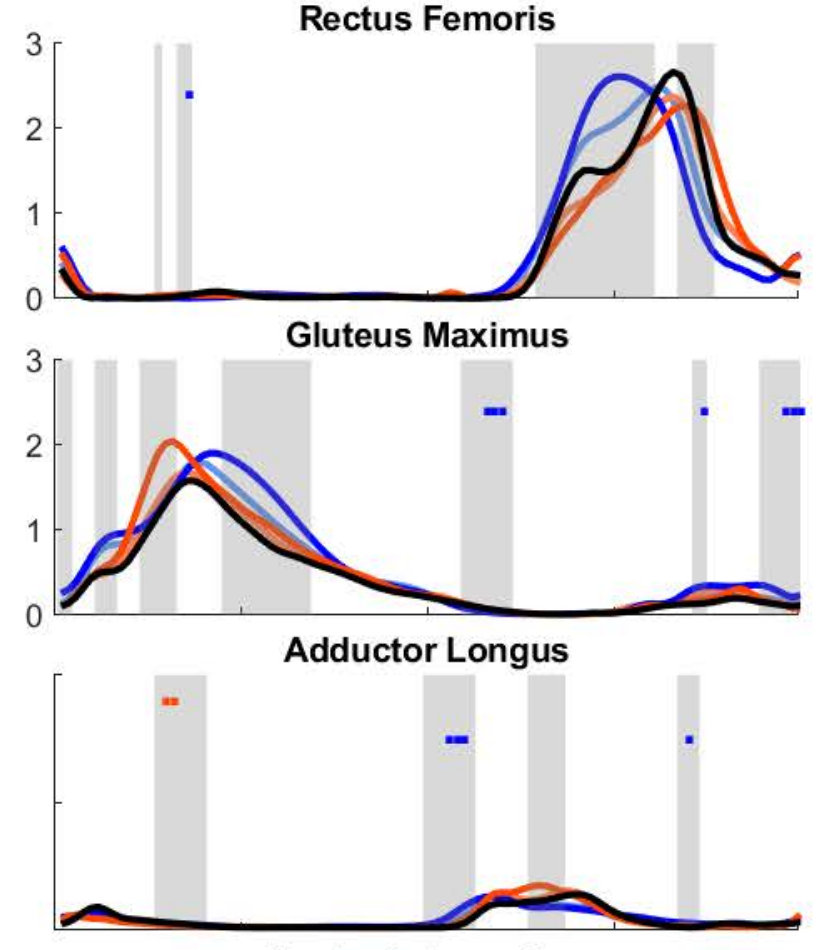

Vastus Intermedius

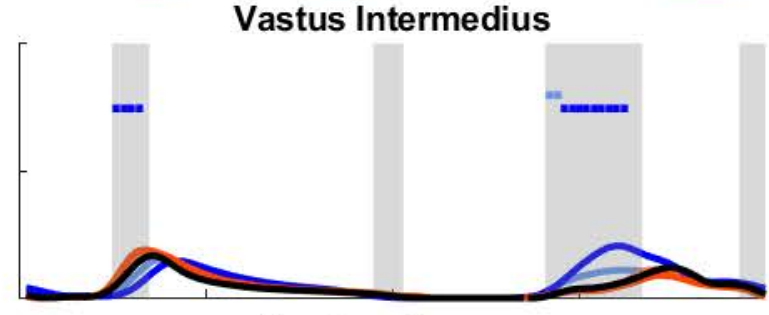

Semitendinosus

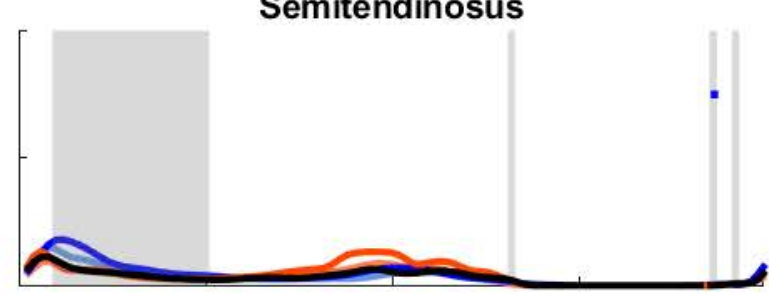

Extensor Digitorum
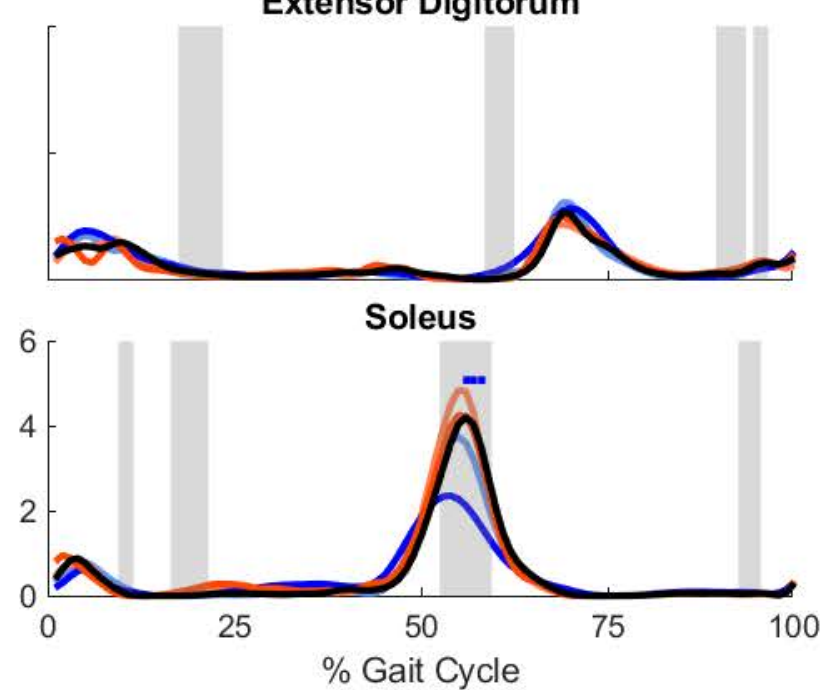

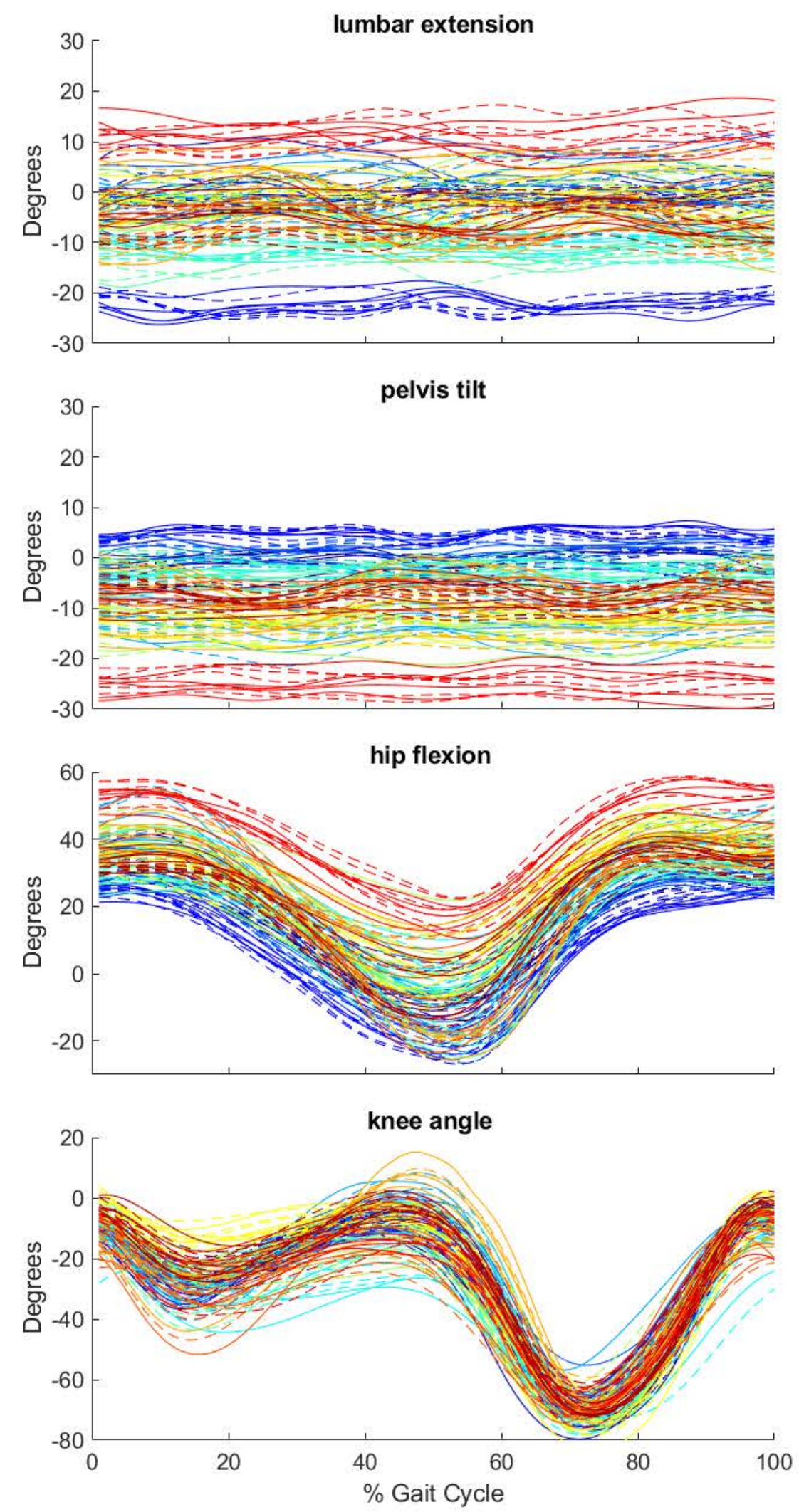
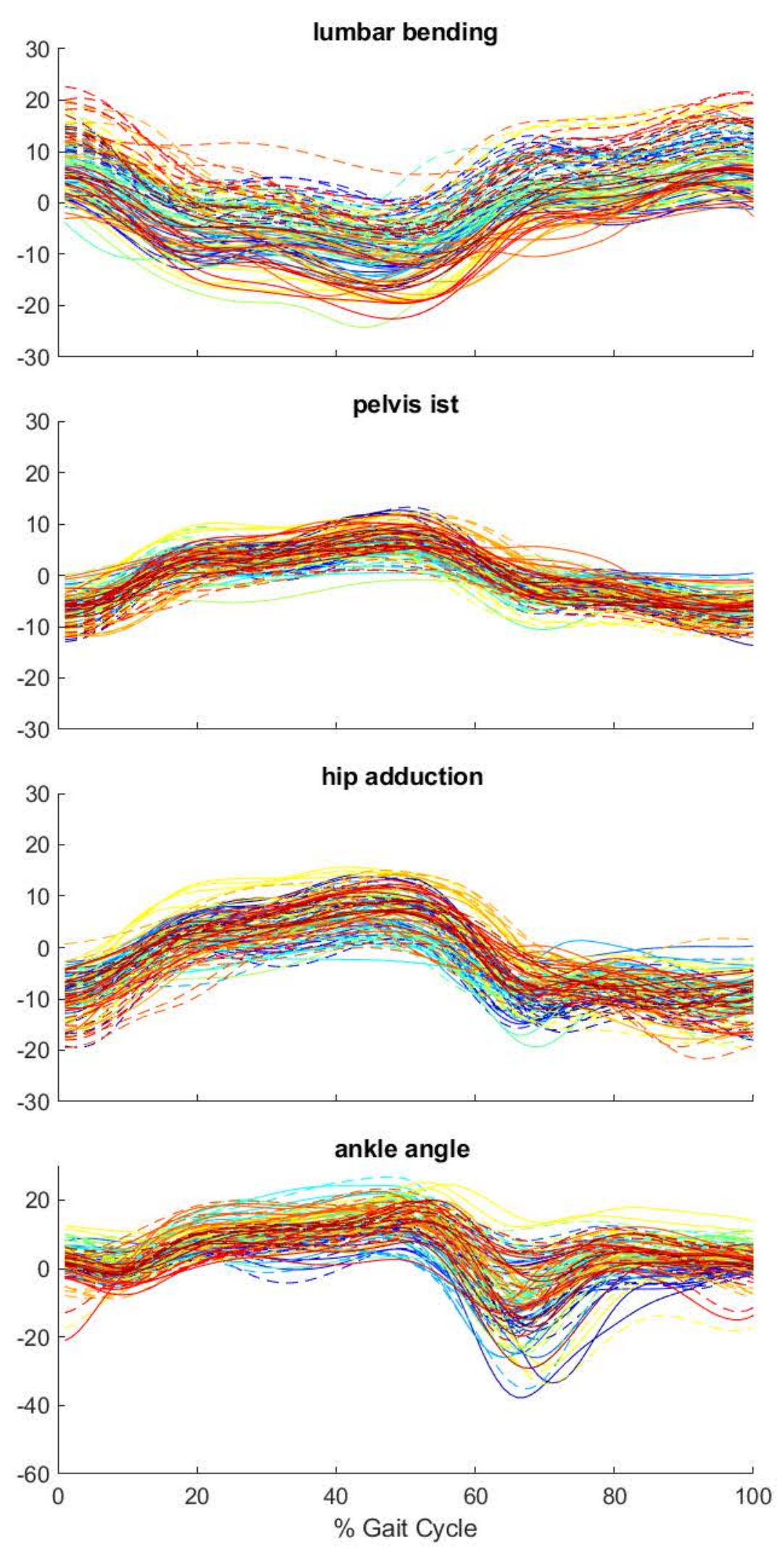
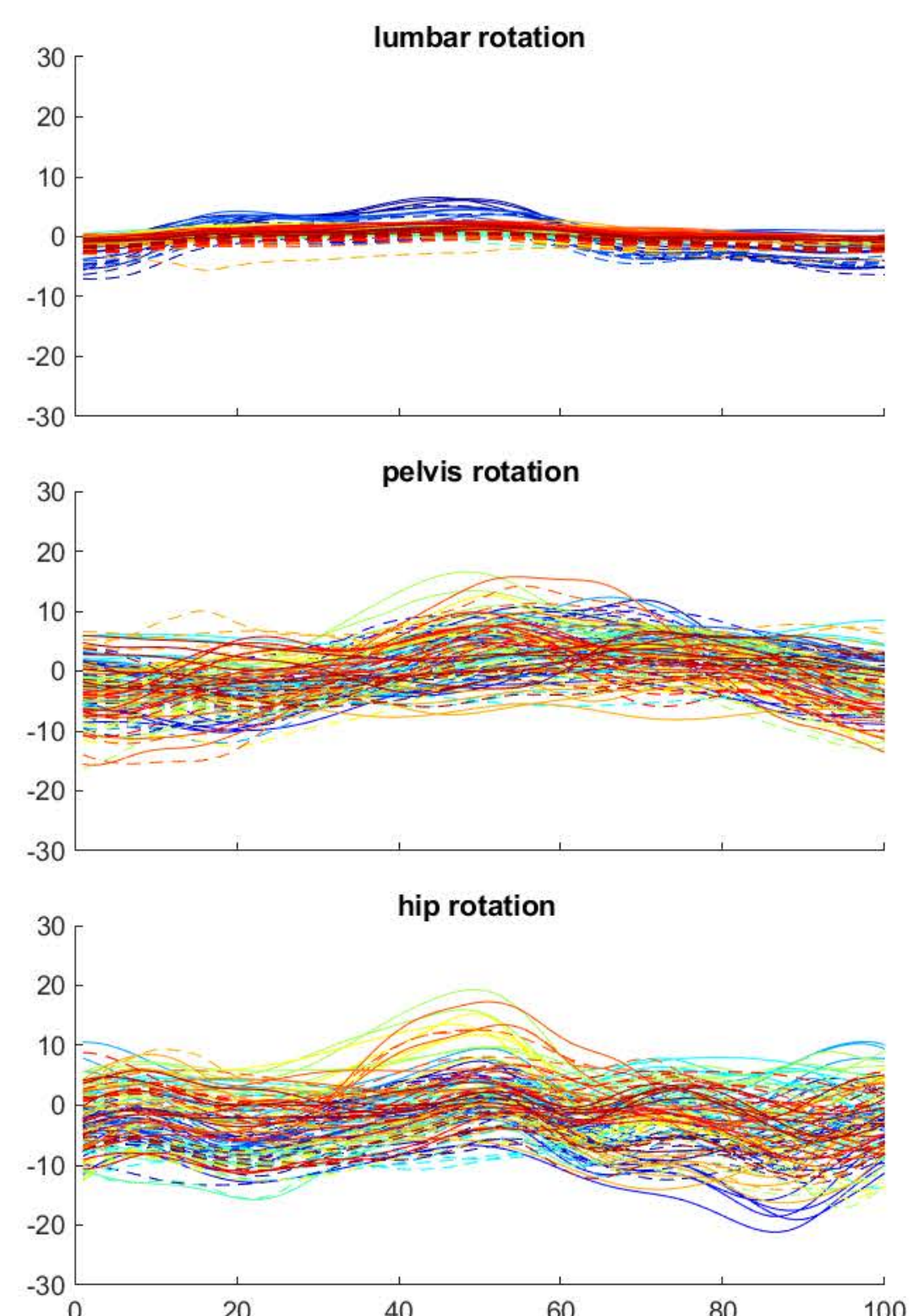

20

YA12

YA11

YA09
YA08
YA06

YA06
YA05

-Left --Right

YA03

YA02

YA01 


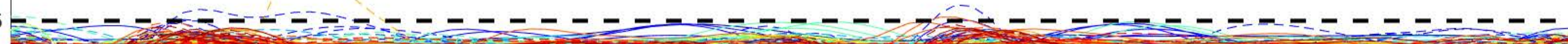

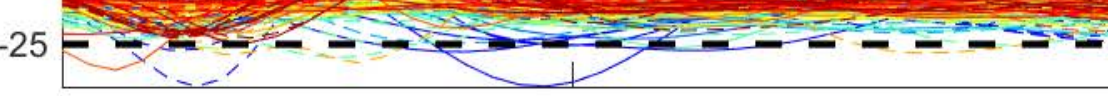

\section{Vertical Forces}

$z_{-25}$

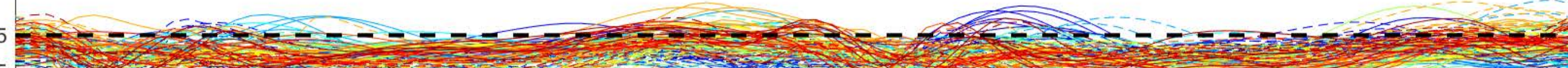

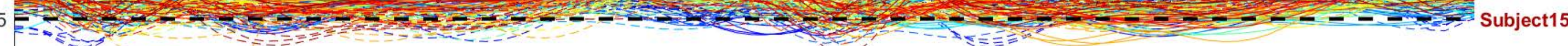

Medial-Lateral Forces

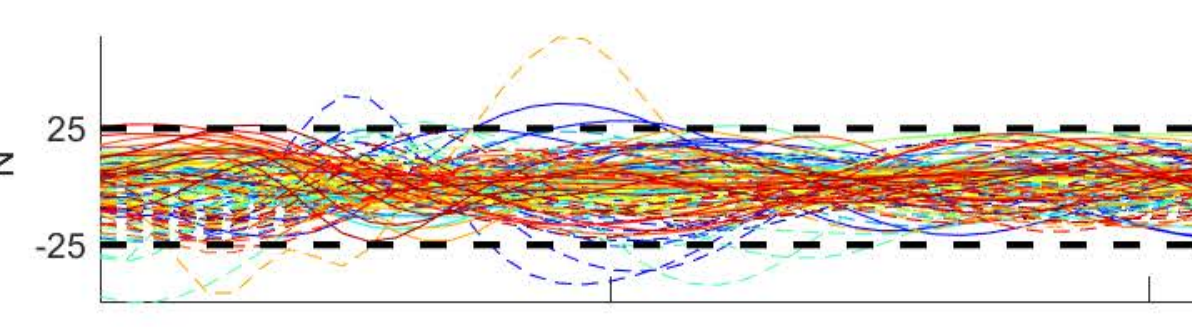

Medial-Lateral Forces

Subject11

Z

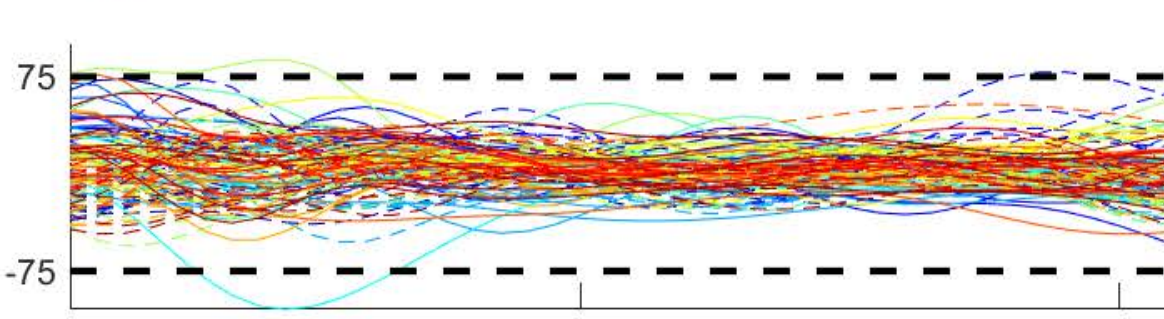

\section{Frontal Plane Moments}

\section{z}

$-75------1---1$

\section{Transverse Plane Moments}

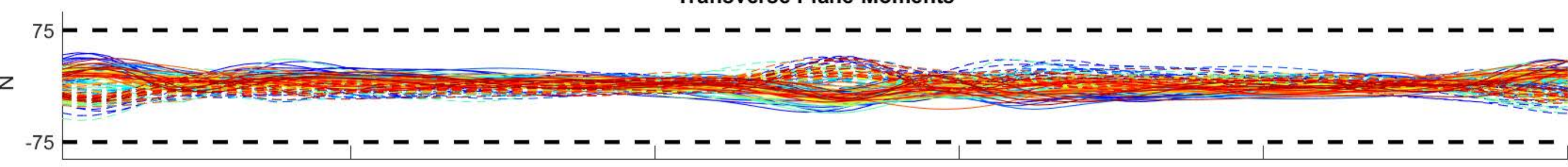

Sagittal Plane Moments
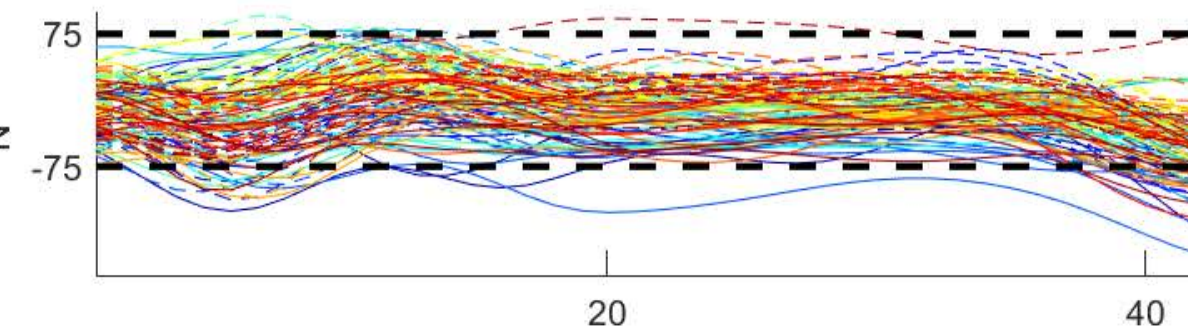

40

$\%$ of Gait Cycle Subject02 Subject01 -- Right - Left

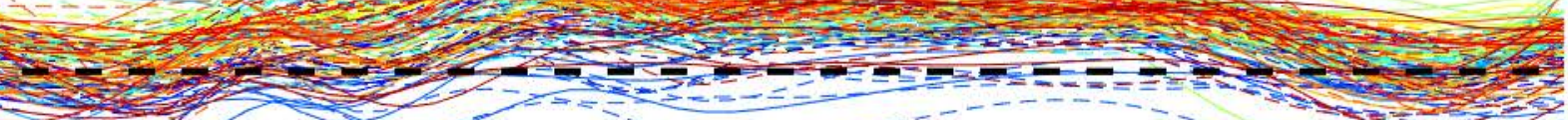




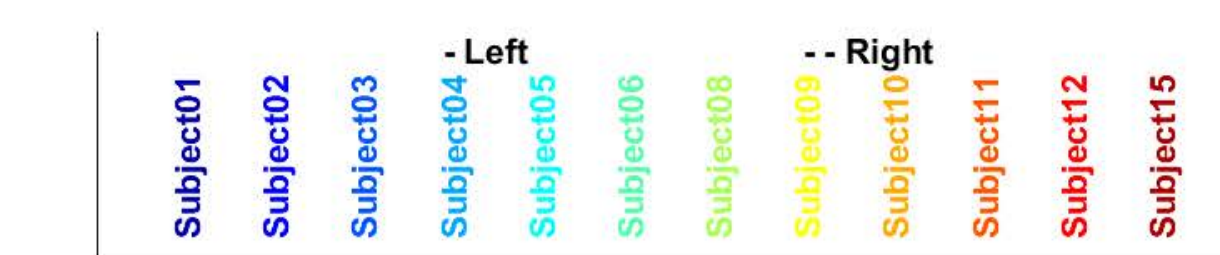

Lumbar Bending

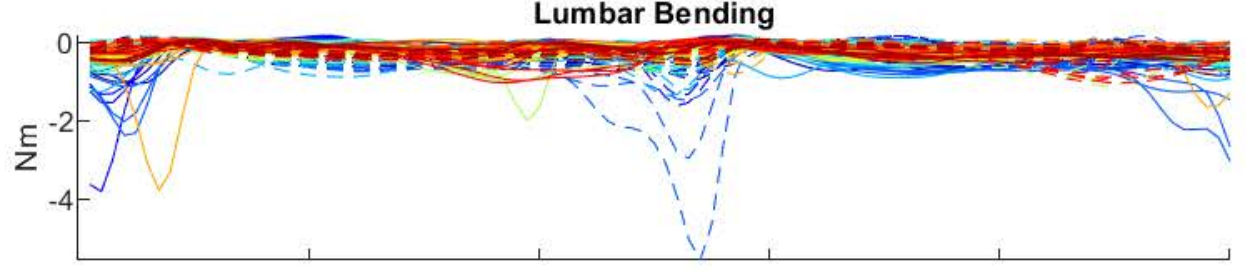

Hip Flexion Left

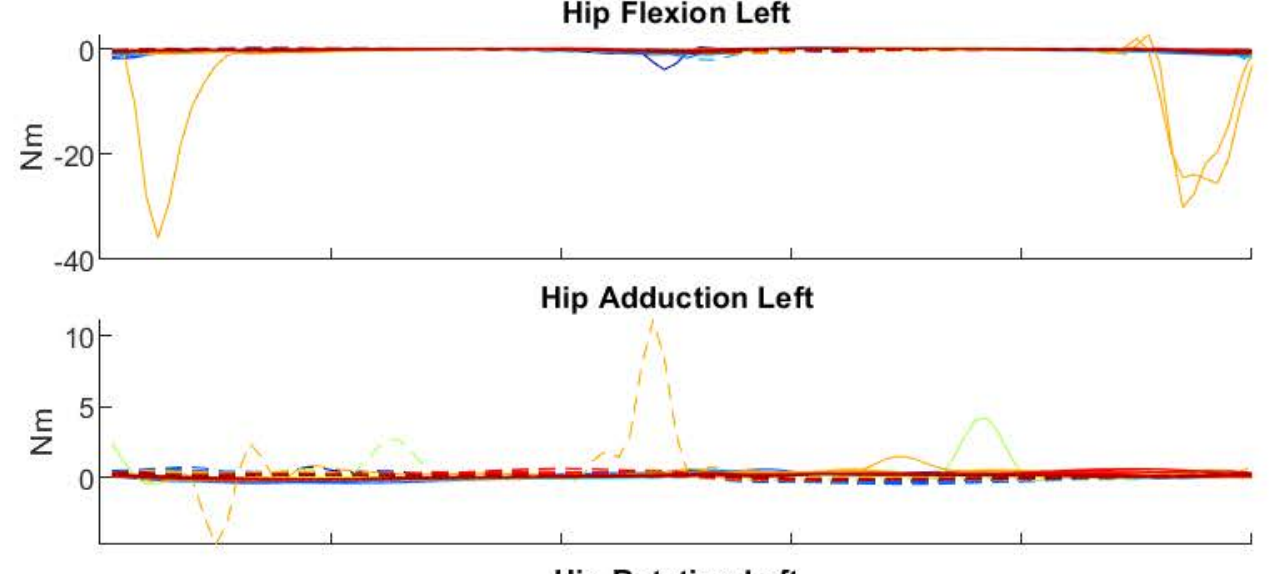

Hip Rotation Left
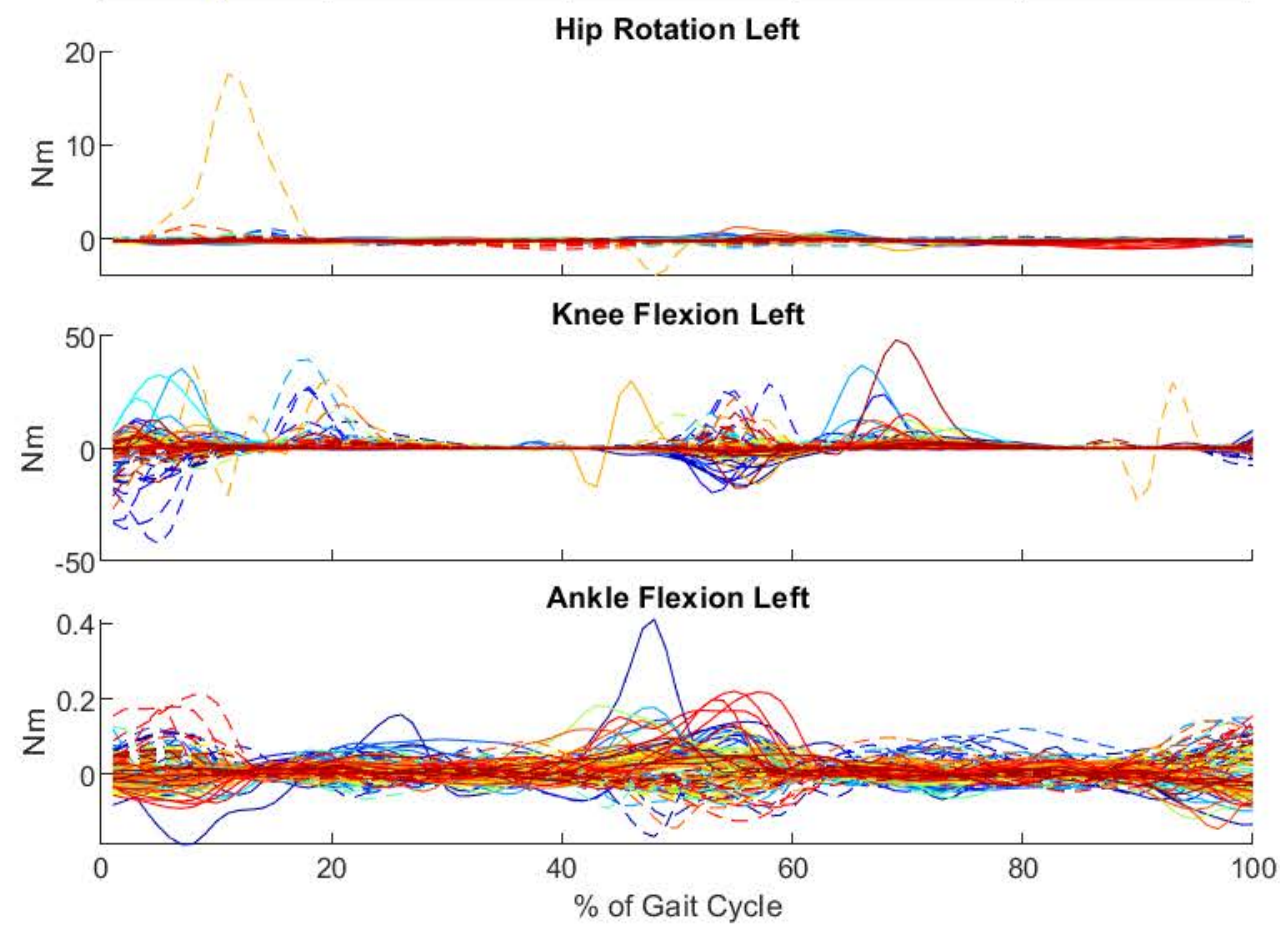

Lumbar Extension

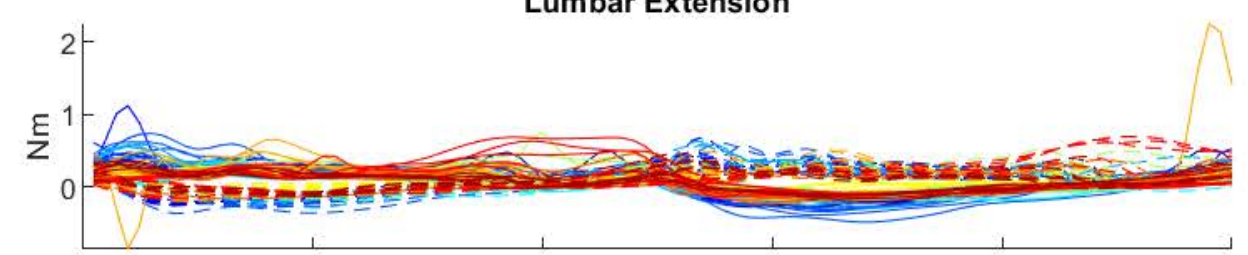

Lumbar Rotation
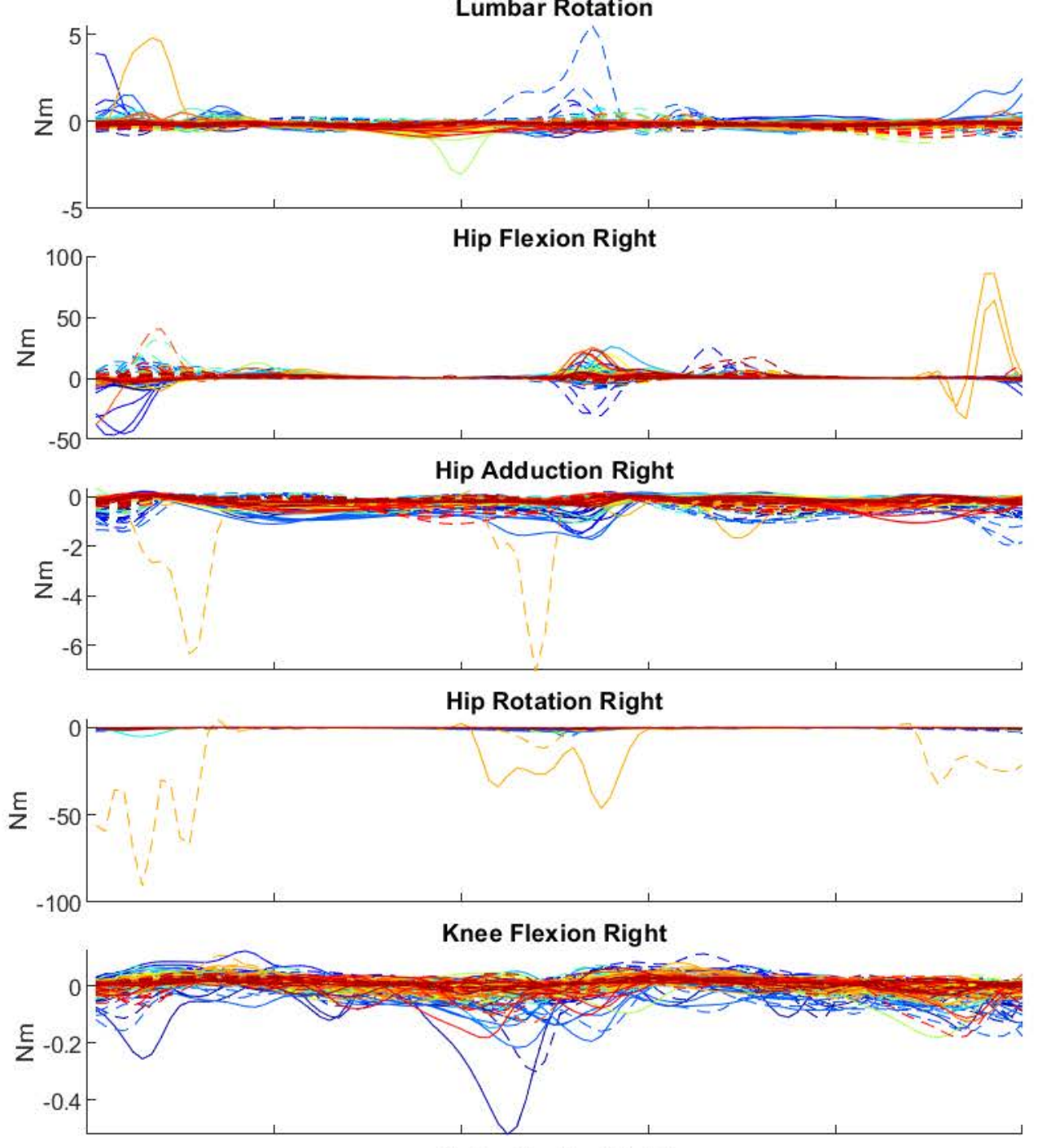

Ankle Flexion Right

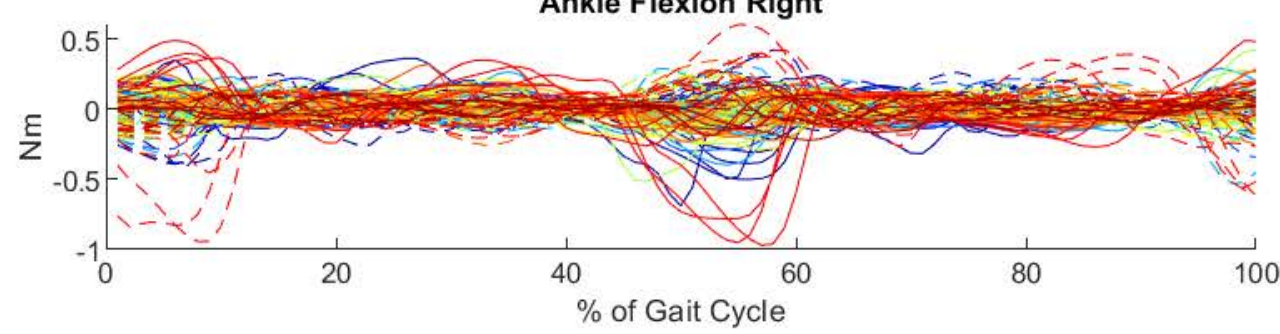



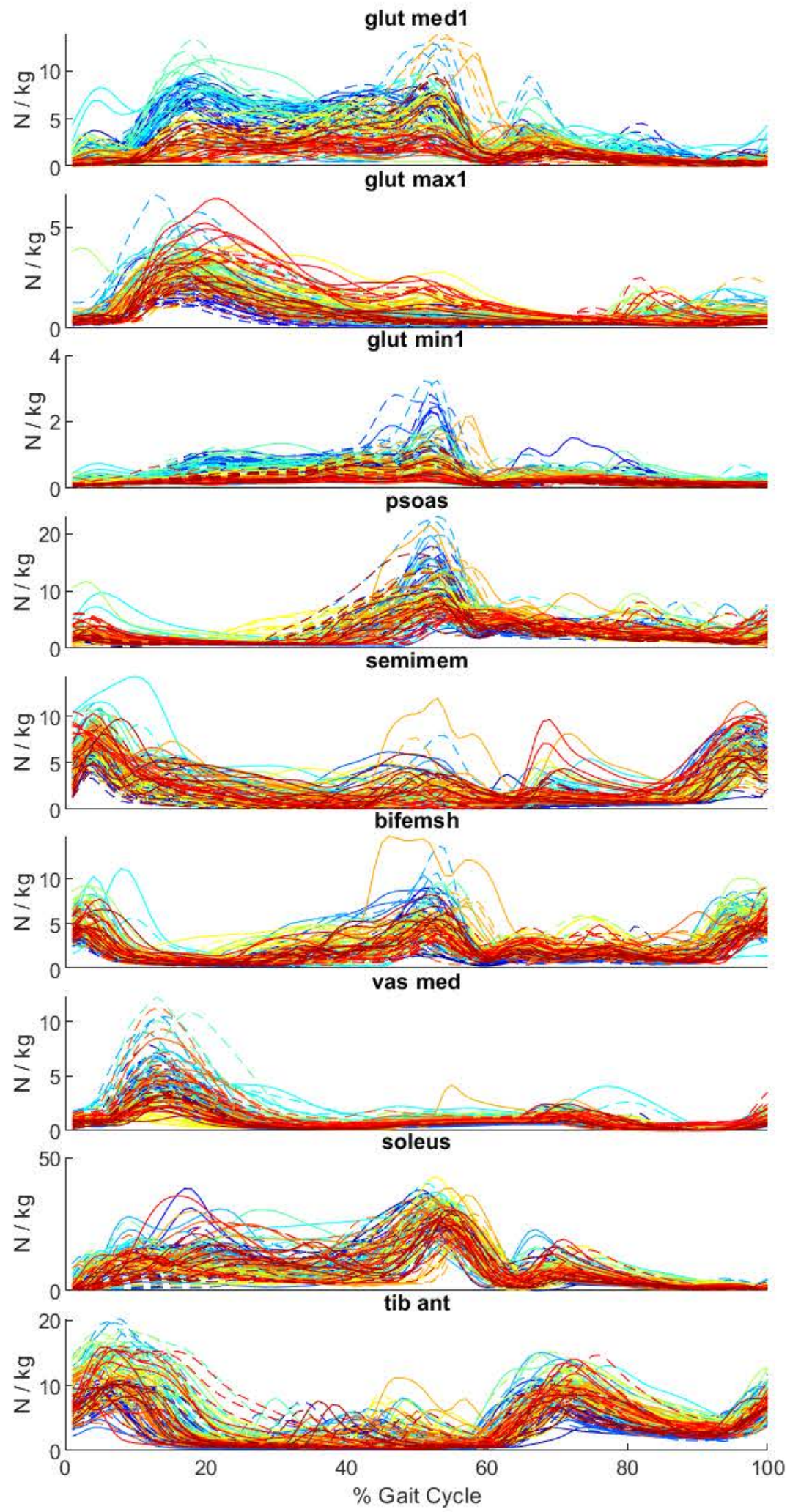
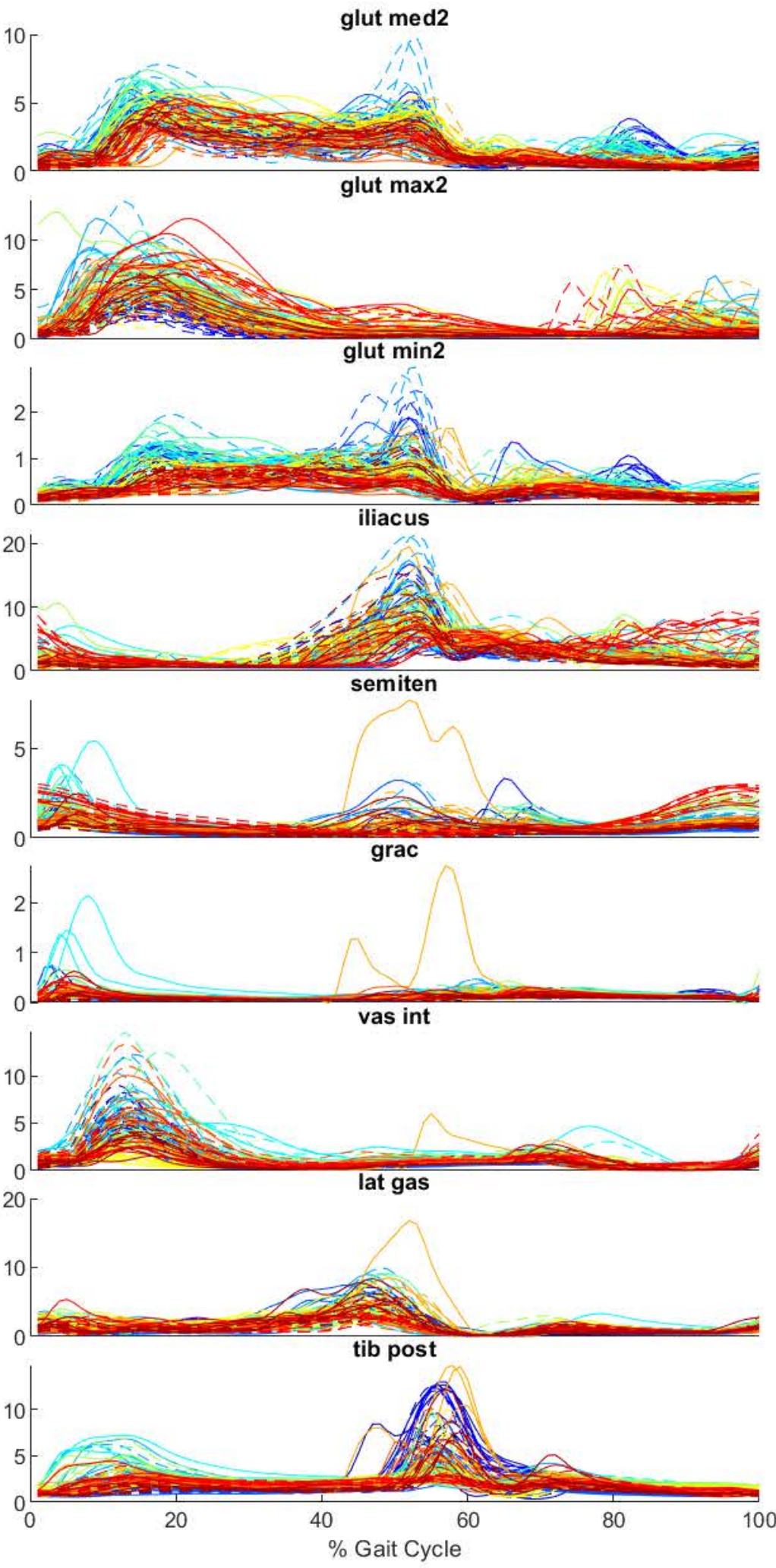

glut med3
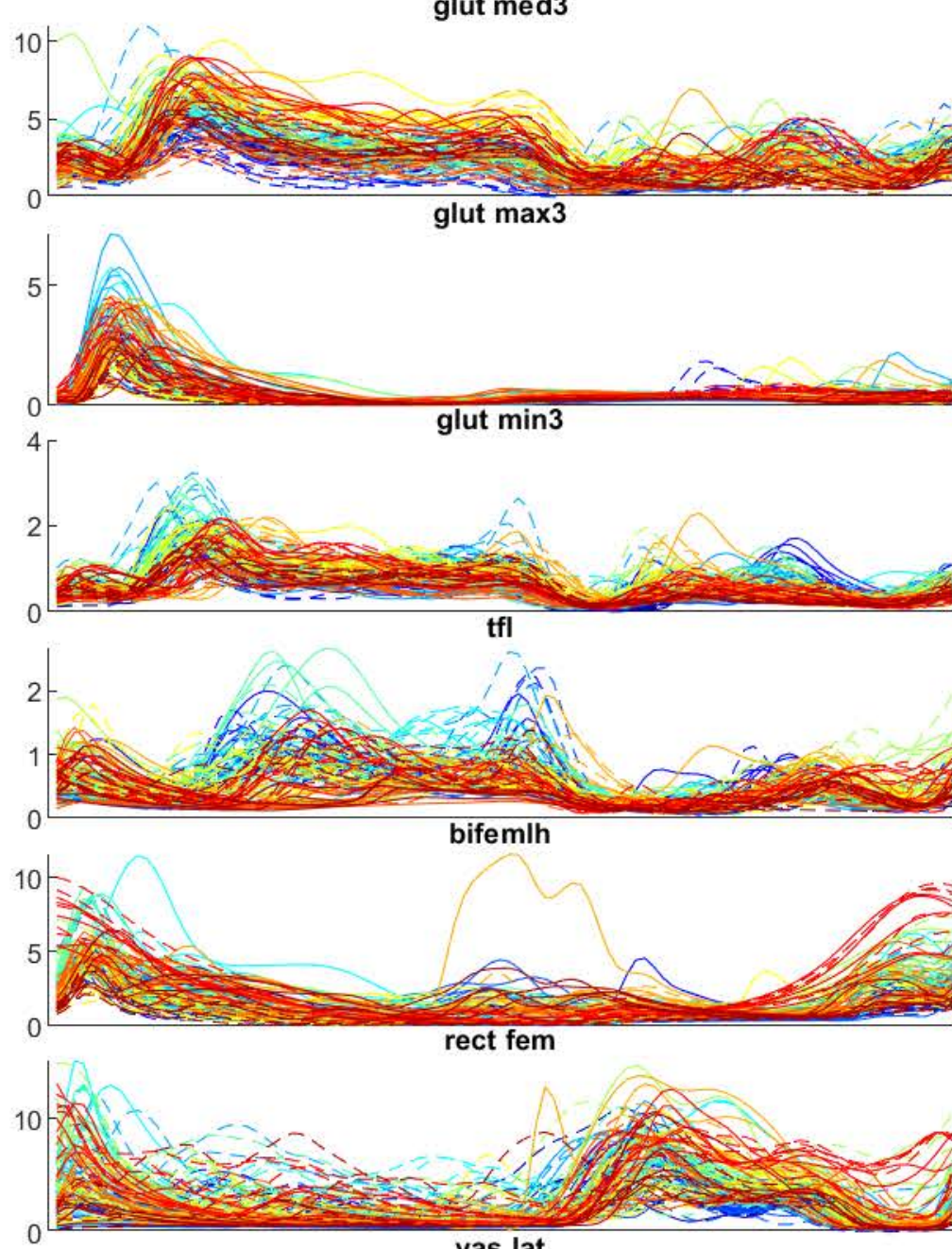

YA11

YA10
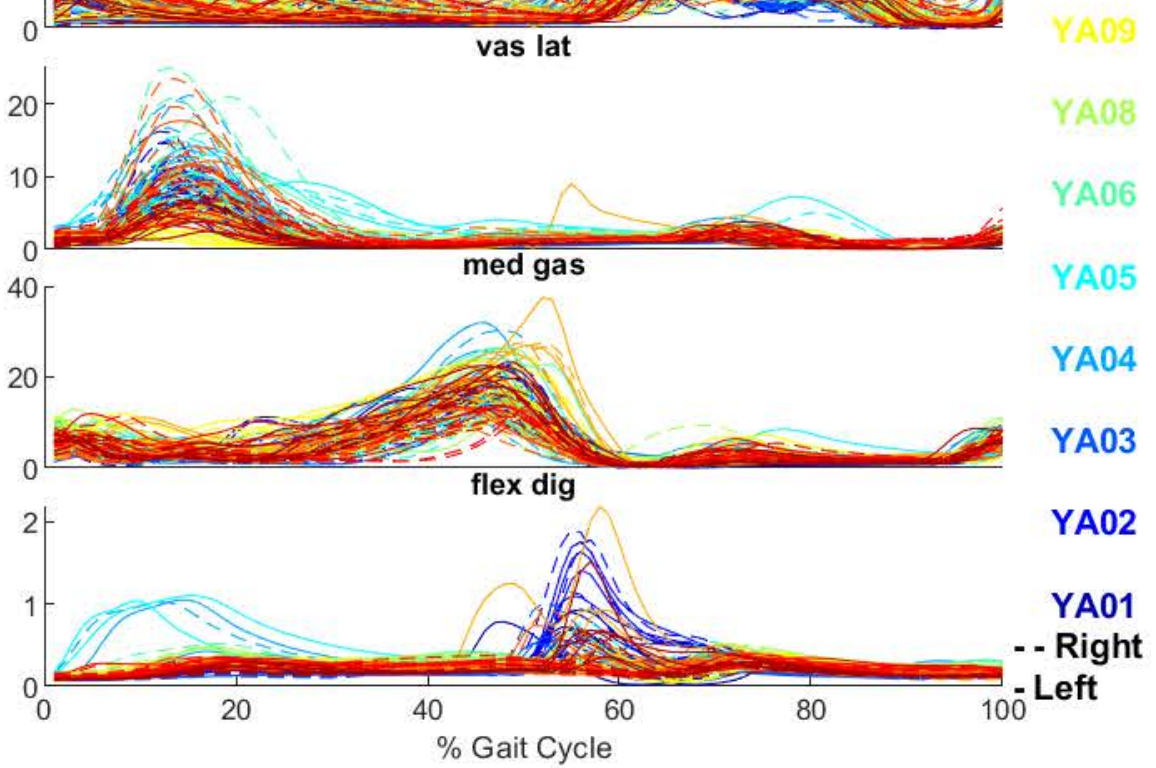

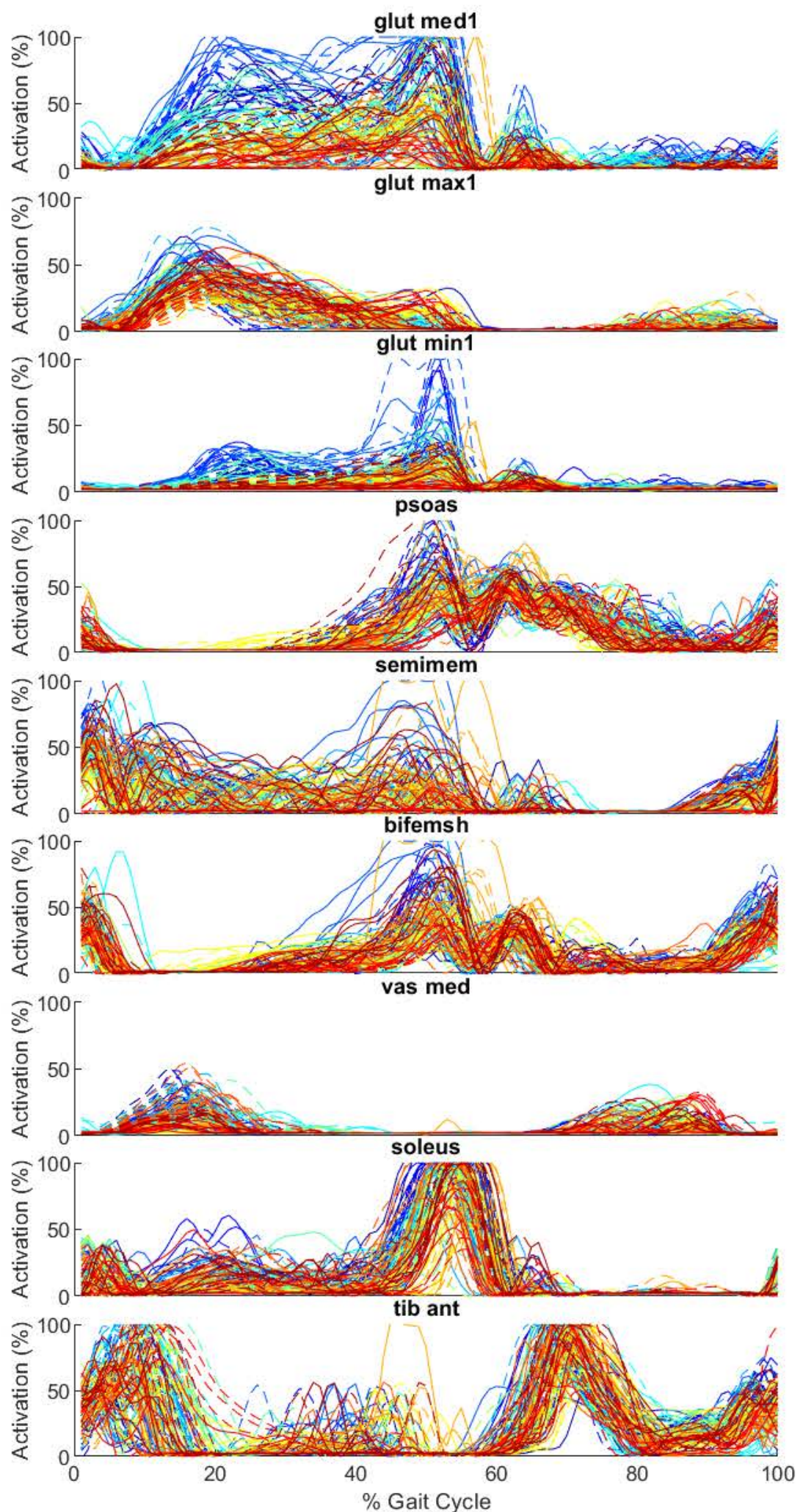

\% Gait Cycle
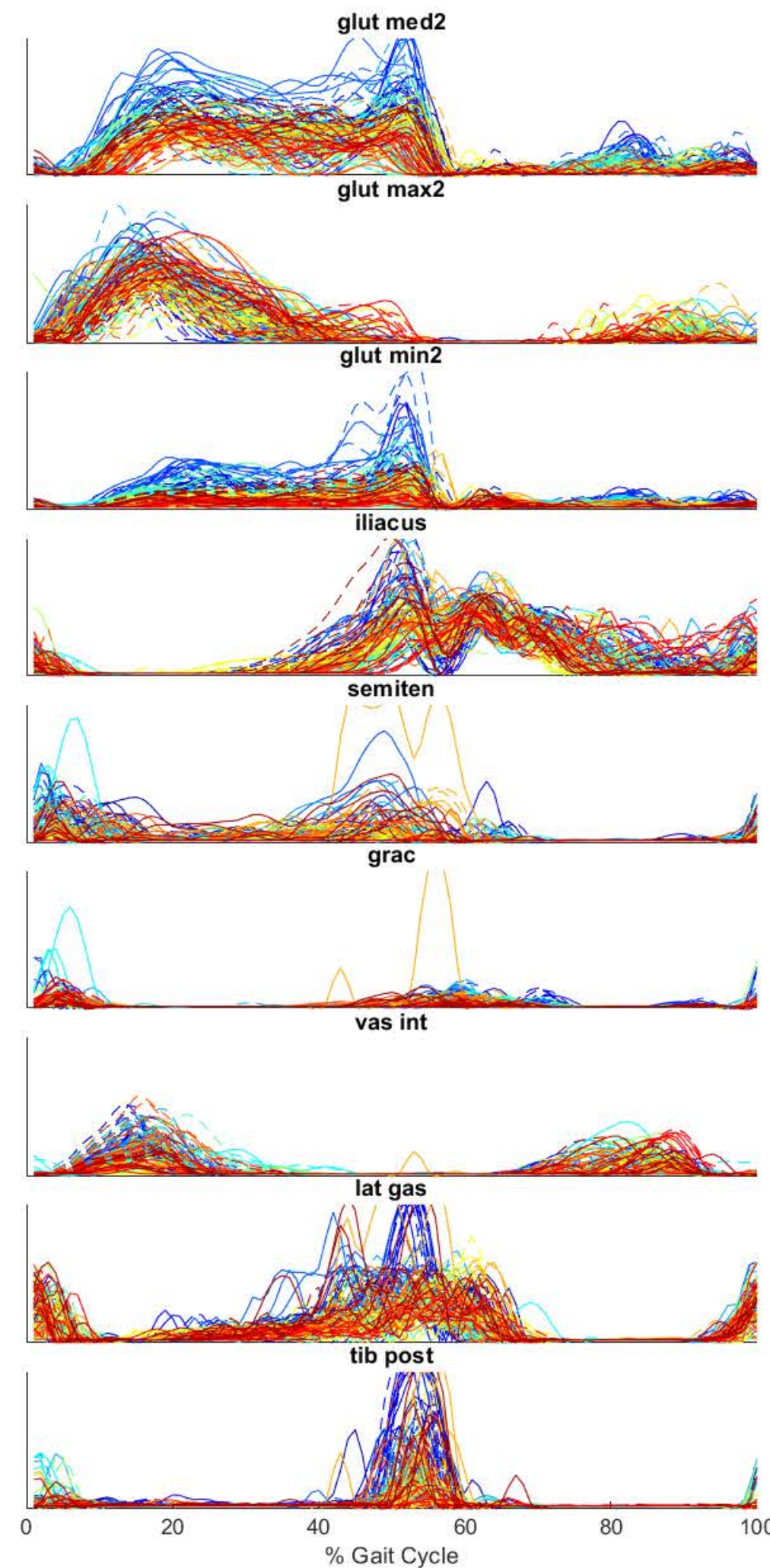

glut med3

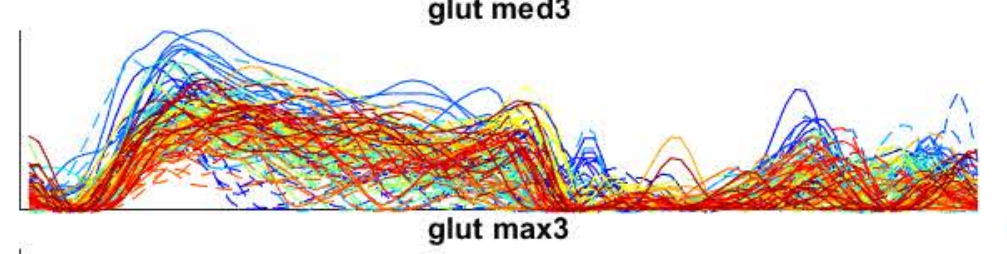

glut $\max 3$

YA15

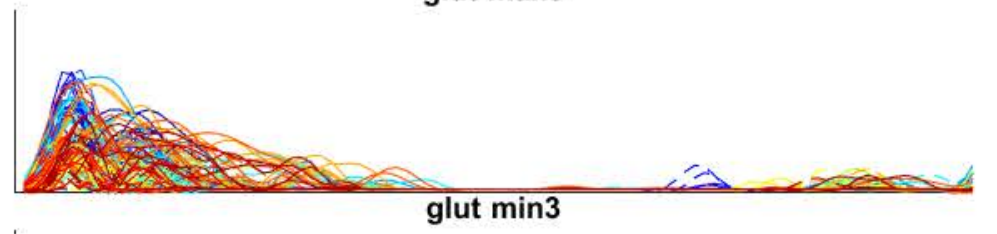

YA12

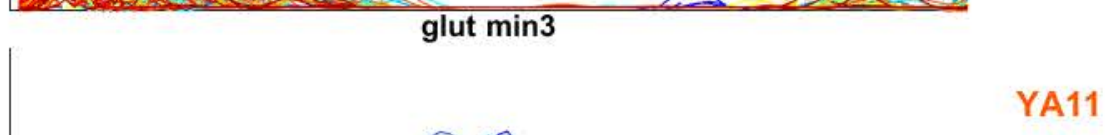

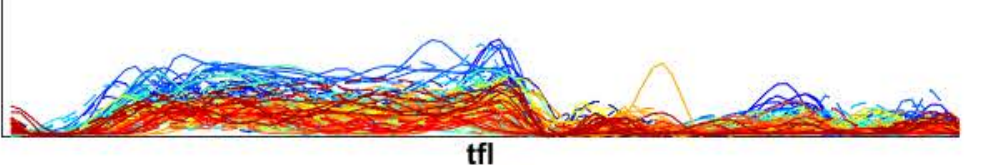
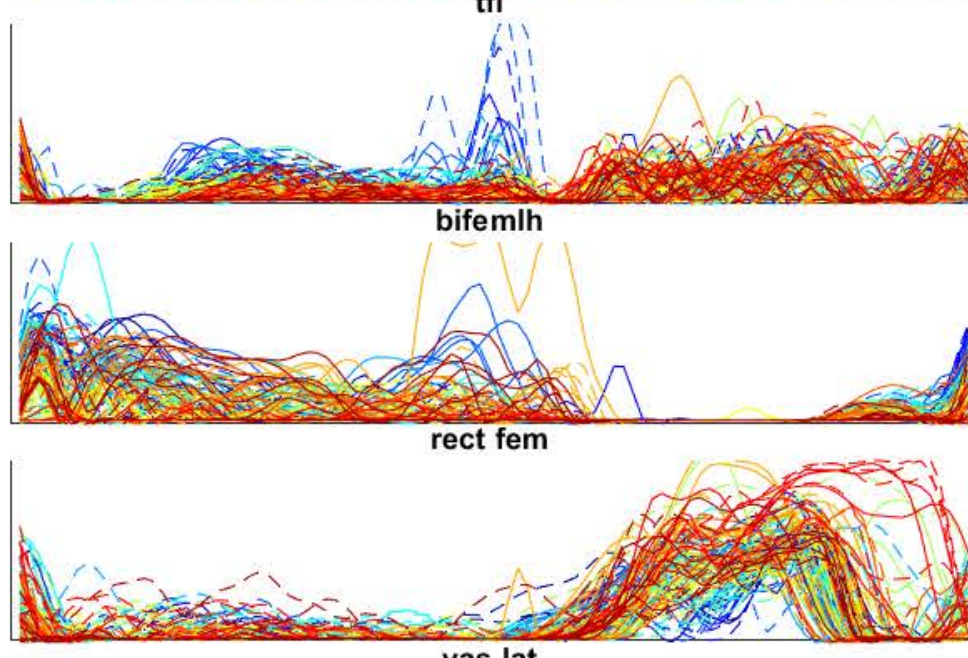

vas lat
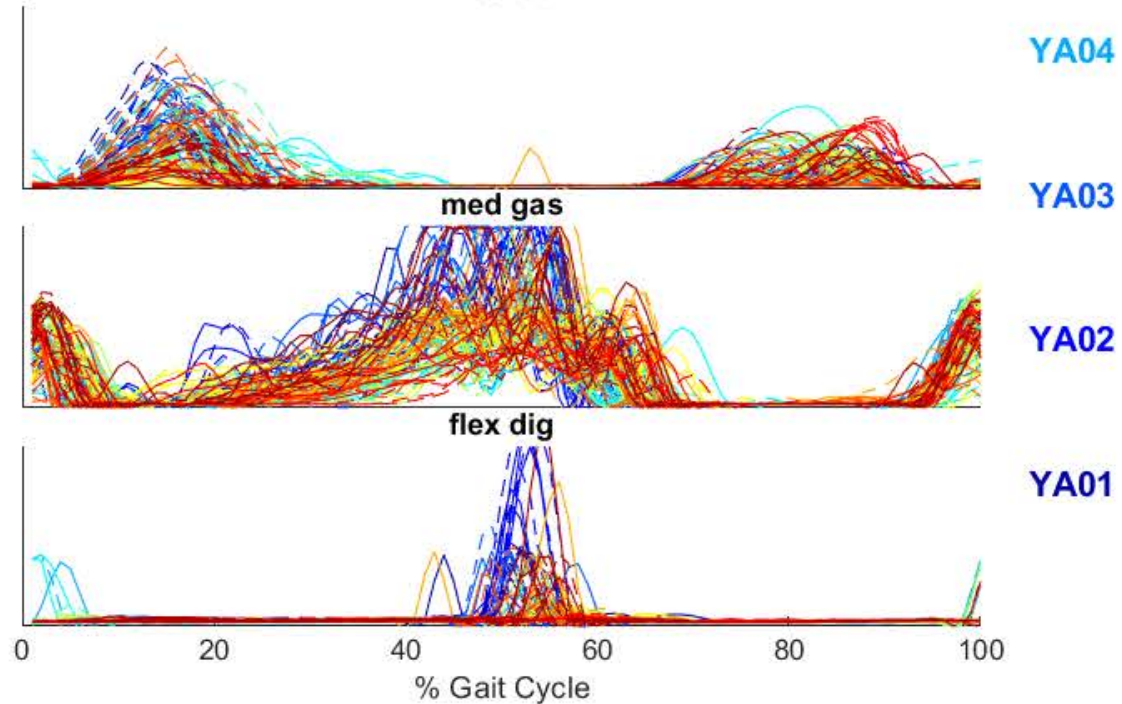


\section{MUSCLE FORCES}
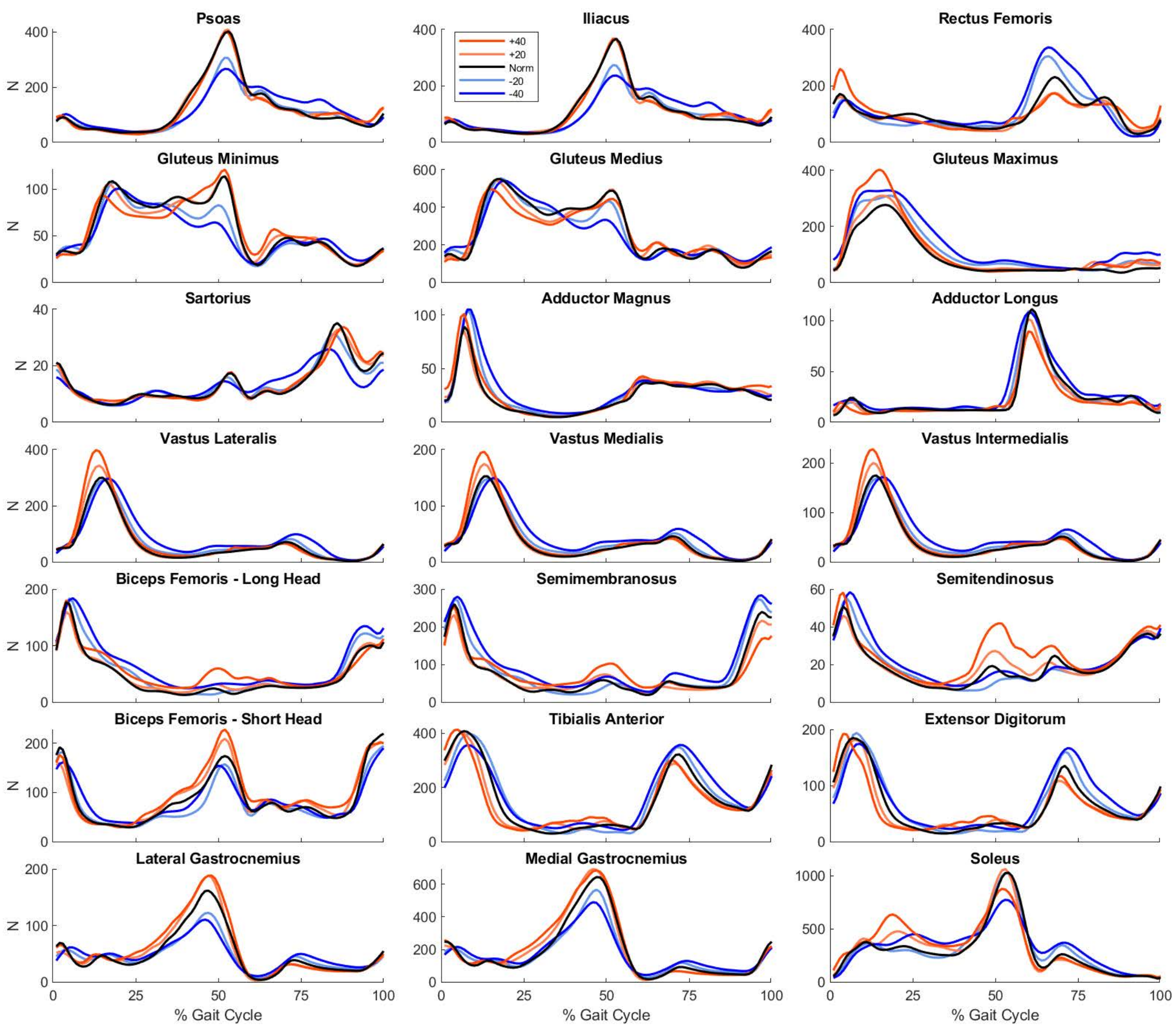


\section{MUSCLE ACTIVATIONS}
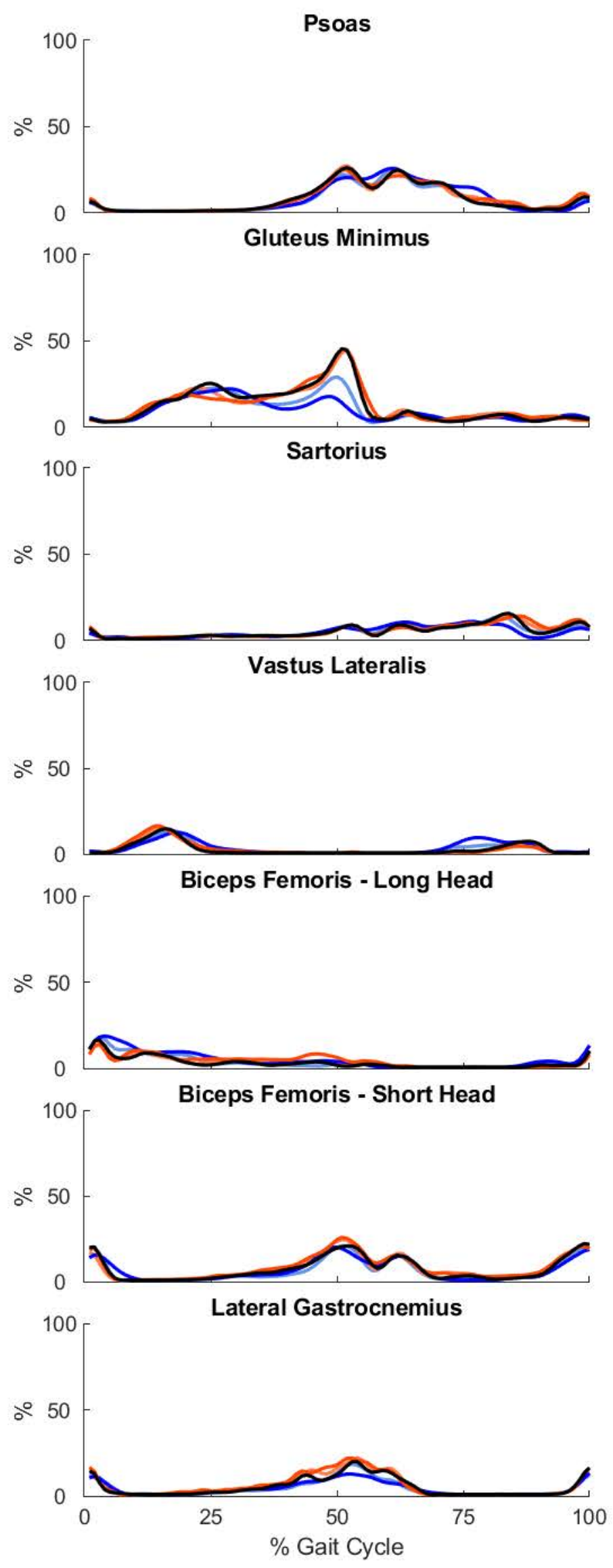

lliacus

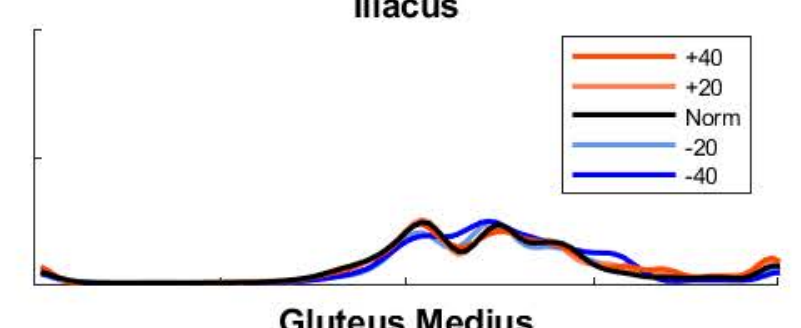

Gluteus Medius
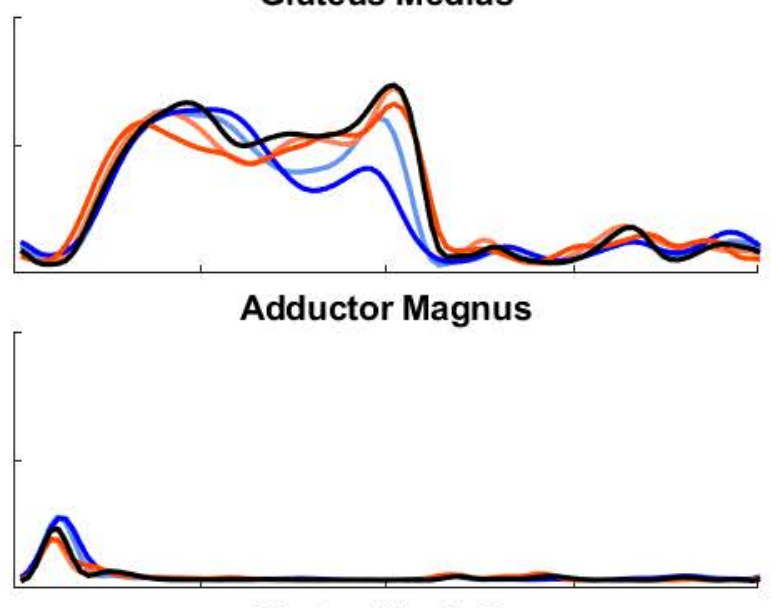

Vastus Medialis
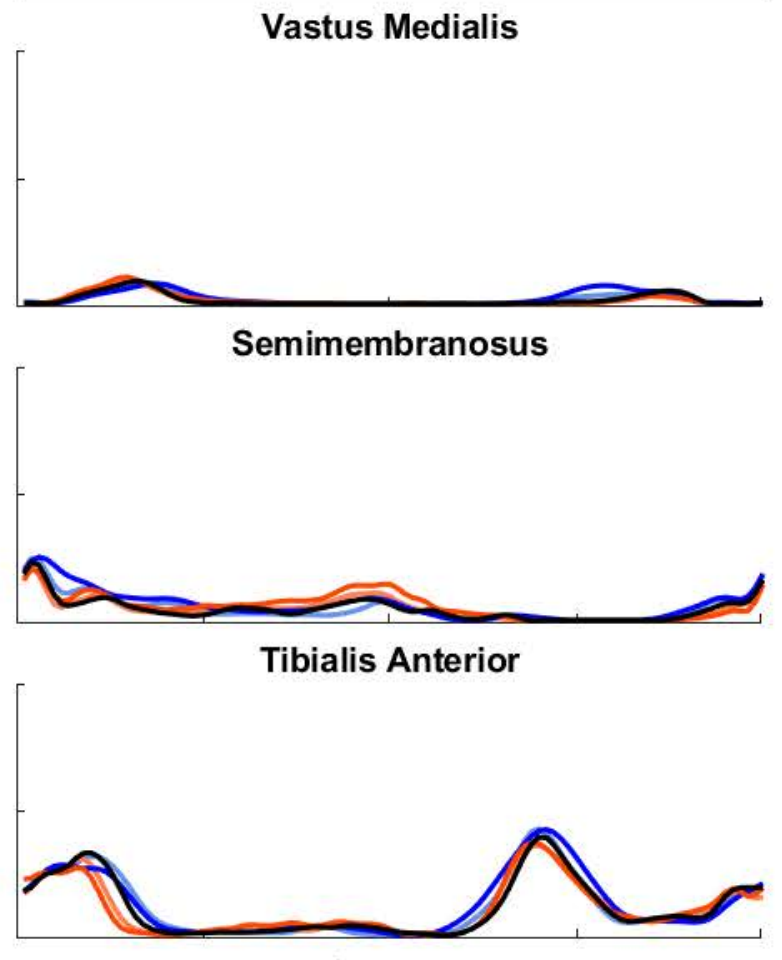

Medial Gastrocnemius

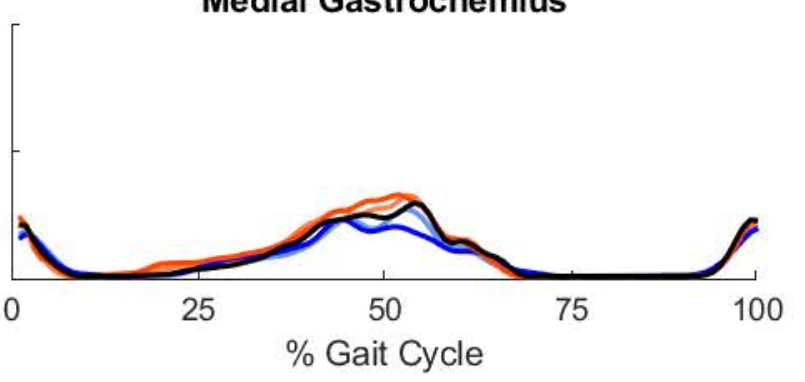

Rectus Femoris

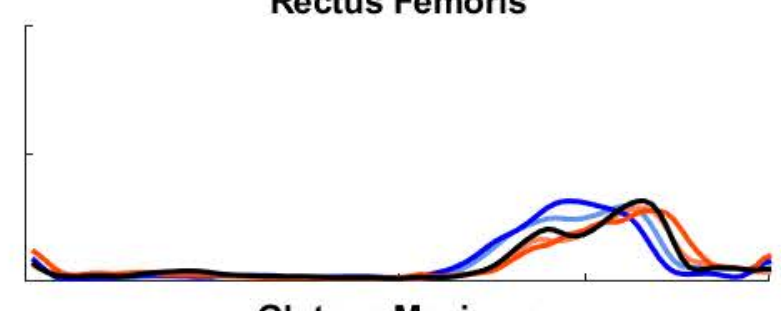

Gluteus Maximus

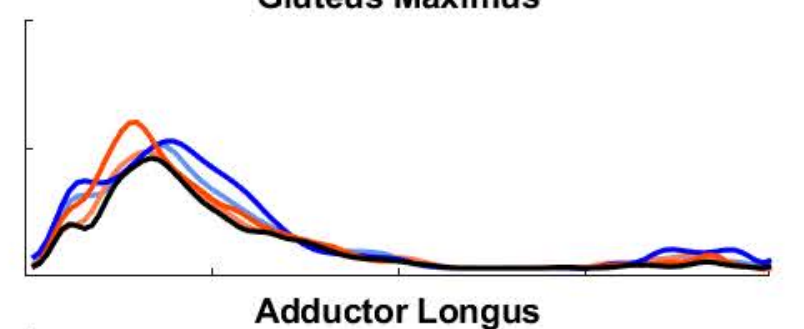

Adductor Longus

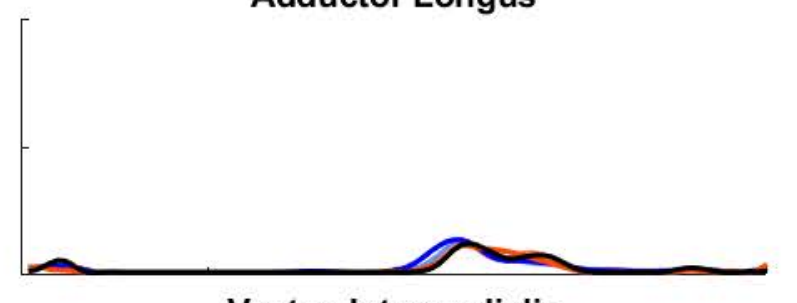

Vastus Intermedialis
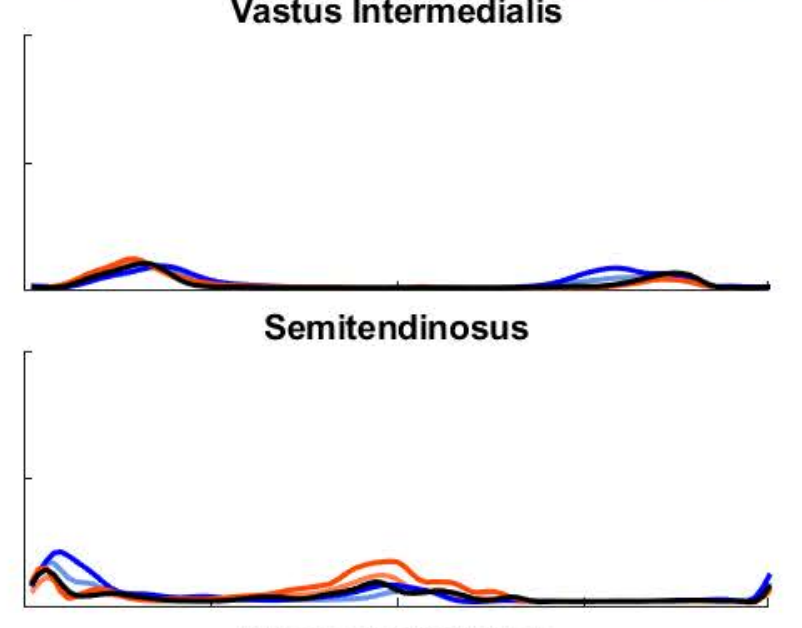

Extensor Digitorum

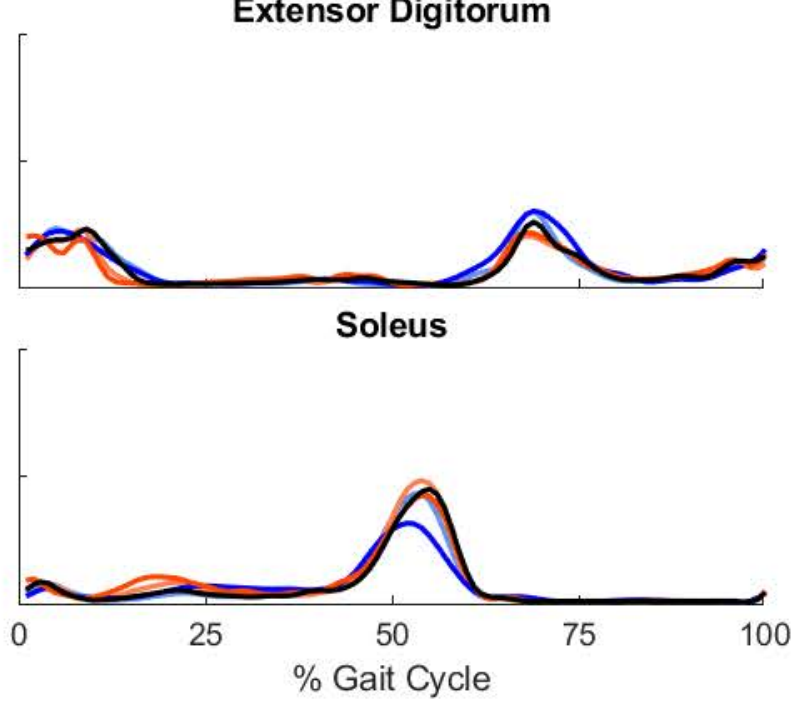

